\title{
RAMANUJAN SUMS VIA GENERALIZED MÖBIUS FUNCTIONS AND APPLICATIONS
}

\author{
VICHIAN LAOHAKOSOL, PATTIRA RUENGSINSUB, AND NITTIYA PABHAPOTE
}

Received 22 May 2006; Revised 20 August 2006; Accepted 5 September 2006

A generalized Ramanujan sum (GRS) is defined by replacing the usual Möbius function in the classical Ramanujan sum with the Souriau-Hsu-Möbius function. After collecting basic properties of a GRS, mostly containing existing ones, seven aspects of a GRS are studied. The first shows that the unique representation of even functions with respect to GRSs is possible. The second is a derivation of the mean value of a GRS. The third establishes analogues of the remarkable Ramanujan's formulae connecting divisor functions with Ramanujan sums. The fourth gives a formula for the inverse of a GRS. The fifth is an analysis showing when a reciprocity law exists. The sixth treats the problem of dependence. Finally, some characterizations of completely multiplicative function using GRSs are obtained and a connection of a GRS with the number of solutions of certain congruences is indicated.

Copyright @ 2006 Hindawi Publishing Corporation. All rights reserved.

\section{Introduction and basic definitions}

The classical Ramanujan sum is the arithmetic function of two variables (see $[2,15,21]$ )

$$
c(n, k)=\sum_{\substack{m(\bmod k) \\ \operatorname{gcd}(m, k)=1}} e^{2 \pi i m n / k},
$$

where $k \in \mathbb{N}, n \in \mathbb{N}_{0}:=\mathbb{N} \cup\{0\}$. Ramanujan sums are closely connected with the usual Möbius function via the identity

$$
c(n, k)=\sum_{d \mid \operatorname{gcd}(n, k)} d \mu\left(\frac{k}{d}\right) .
$$

Recently, there have been a great deal of interest in extending results (see $[8,14,24])$ related to the classical Möbius function to those of a generalized Möbius function, also 
known as the Souriau-Hsu-Möbius function, $\mu_{\alpha}$, which is defined by [3],

$$
\mu_{\alpha}(n)=\prod_{p \mid n}\left(\begin{array}{c}
\alpha \\
v_{p}(n)
\end{array}\right)(-1)^{v_{p}(n)}
$$

where $\alpha \in \mathbb{C}$, and $n=\prod p^{v_{p}(n)}$ denotes the unique prime factorization of $n \in \mathbb{N}, v_{p}(n)$ being the largest exponent of the prime $p$ that divides $n$.

Our objectives here are

(1) to define the generalized Ramanujan sum, abbreviated as GRS, by replacing the usual Möbius function with the Souriau-Hsu-Möbius function and to derive their arithmetical properties extending the known ones;

(2) to investigate whether the set of GRSs can be used as a basis for expanding even functions;

(3) to derive the mean value of a GRS;

(4) to derive analogues of Ramanujan's remarkable formulae relating the divisor functions with sums of GRSs;

(5) to derive the Dirichlet inverse of a GRS using Haukkanen's idea (see [9]) of introducing principal functions and to analyze whether a reciprocity law exists;

(6) to investigate the dependence of GRSs; and lastly,

(7) to characterize completely multiplicative functions using GRSs, extending an earlier work of Ivić (see [12]), and to indicate a connection of a GRS with the number of solutions of certain congruences.

Let us begin by recalling [2, Theorem 8.5, page 161]: if

$$
S_{k}(n)=\sum_{d \mid \operatorname{gcd}(n, k)} f(d) g\left(\frac{k}{d}\right)
$$

then $S_{k}(n)$ has a finite Fourier expansion of the form

$$
S_{k}(n)=\sum_{m(\bmod k)} a_{k}(m) e^{2 \pi m n i / k}
$$

where $a_{k}(m)=\sum_{d \mid \operatorname{gcd}(m, k)} g(d) f(k / d)(d / k)$.

Substituting $f(m)=m, g(m)=\mu_{\alpha}(m)$ and using the fact [3, Corollary 2] that $\mu_{\alpha} *$ $\mu_{-1}=\mu_{\alpha-1}$, this simple result leads to the following.

Theorem 1.1. If $k \in \mathbb{N}, n \in \mathbb{N}_{0}$, and $\alpha \in \mathbb{C}$, then

$$
\sum_{d \mid \operatorname{gcd}(n, k)} d \mu_{\alpha}\left(\frac{k}{d}\right)=\sum_{m(\bmod k)} \mu_{\alpha-1}(\operatorname{gcd}(m, k)) e^{2 \pi m n i / k}
$$

We define the generalized Ramanujan sum, GRS, of order $\alpha \in \mathbb{C}$, by

$$
c^{(\alpha)}(n, k):=\sum_{d \mid \operatorname{gcd}(n, k)} d \mu_{\alpha}\left(\frac{k}{d}\right)=\sum_{m(\bmod k)} \mu_{\alpha-1}(\operatorname{gcd}(m, k)) e^{2 \pi m n i / k} \quad\left(n \in \mathbb{N}_{0}, k \in \mathbb{N}\right) .
$$


When $\alpha=1$, we get back to the usual Ramanujan sum, which will always be written without the indication on $\alpha$. The following basic properties will be frequently used and are easily verified, so we omit their proofs.

Lemma 1.2. Let $m \in \mathbb{N}_{0}, n \in \mathbb{N}$, p prime, and $\alpha \in \mathbb{C}$. Then

(1) $\sum_{j \mid n} c^{(\alpha)}(m, j)=c^{(\alpha-1)}(m, n)$,

(2)

$$
c^{(0)}(m, n)= \begin{cases}n & \text { if } n \mid m, \\ 0 & \text { otherwise }\end{cases}
$$

(3) $c^{(-1)}(m, n)=\sigma_{1}(\operatorname{gcd}(m, n))$, where $\sigma_{1}(t)=\sum_{d \mid t} d$,

(4) $c^{(\alpha)}(n, 1)=1$,

(5) $c^{(\alpha)}(1, m)=\mu_{\alpha}(m)$,

(6) $c^{(\alpha)}(n, m)=\mu_{\alpha}(m)$ if $\operatorname{gcd}(n, m)=1$,

(7) $c^{(\alpha)}(a b, m k)=c^{(\alpha)}(a, m) c^{(\alpha)}(b, k)$ whenever $\operatorname{gcd}(a, k)=\operatorname{gcd}(b, m)=\operatorname{gcd}(m, k)=1$,

(8) $c^{(\alpha)}(a b, m)=c^{(\alpha)}(a, m)$ if $\operatorname{gcd}(b, m)=1$,

(9) $c^{(\alpha)}(a, m k)=c^{(\alpha)}(a, m) \mu_{\alpha}(k)$ if $\operatorname{gcd}(a, k)=\operatorname{gcd}(m, k)=1$,

(10) $c^{(\alpha)}(n, r s)=c^{(\alpha)}(n, r) c^{(\alpha)}(n, s)$ if $\operatorname{gcd}(r, s)=1$,

(11) for $a, b \in \mathbb{N}, c^{(\alpha)}\left(p^{b}, p^{a}\right)=\sum_{i=0}^{\min (a, b)}(-1)^{a-i}\left(\begin{array}{c}\alpha \\ a-i\end{array}\right) p^{i}, c^{(\alpha)}\left(p^{b}, p\right)=p-\alpha$,

(12) $c^{(\alpha)}\left(n, p^{a}\right)=(-1)^{a}\left(\begin{array}{l}\alpha \\ a\end{array}\right)$ if $p \nmid n$,

(13) $c^{(\alpha)}\left(n, p^{a}\right)=\sum_{i=0}^{\min (a, k)}(-1)^{a-i}\left(\begin{array}{c}\alpha \\ a-i\end{array}\right) p^{i}$ if $p^{k} \| n$.

\section{More properties}

In this section, we derive many more properties about GRSs, similar to those in the beginning of [15, Chapter 2] and [2, Chapter 8]. Throughout this section, assume that $g$ is a multiplicative function and $h$ a completely multiplicative function. For $\alpha \in \mathbb{C}, n \in \mathbb{N}_{0}$, $r \in \mathbb{N}$, define

$$
\begin{aligned}
f_{\alpha}(n, r) & :=\sum_{d \mid \operatorname{gcd}(n, r)} h(d) g\left(\frac{r}{d}\right) \mu_{\alpha}\left(\frac{r}{d}\right), \\
F_{\alpha}(r) & :=f_{\alpha}(0, r)=\left(h * g \mu_{\alpha}\right)(r) .
\end{aligned}
$$

Recall that a positive integer is said to be $d$-powerful, $d \in \mathbb{N}$, if it is divisible by each of its prime factors up to a power of $d$. It is easily checked that

(1) $F_{\alpha}(r)$ is a multiplicative function of $r$;

(2) if $r \mid n$, then $F_{\alpha}(r)=f_{\alpha}(0, r)=f_{\alpha}(n, r)$;

(3) let $\zeta_{k}(n)=n^{k}$; for $h(n)=\zeta_{1}(n)$ and $g(n)=\zeta_{0}(n)$, we have [24],

$$
c^{(\alpha)}(0, r)=\sum_{d \mid r} d \mu_{\alpha}\left(\frac{r}{d}\right)=\left(\zeta_{1} * \mu_{\alpha}\right)(r)=r \prod_{p \mid r} \sum_{i=0}^{v_{p}(r)}\left(\begin{array}{c}
\alpha \\
i
\end{array}\right)(-p)^{-i} .
$$


4 Ramanujan sums via generalized Möbius functions

In particular, if $\alpha \in \mathbb{N}$ and $r$ is $\alpha$-powerful, this simplifies to

$$
c^{(\alpha)}(0, r)=r \prod_{p \mid r}\left(1-\frac{1}{p}\right)^{\alpha} .
$$

From [24], an Euler-type totient is defined as an arithmetic function of the form

$$
\phi_{k}^{(\alpha)}:=\zeta_{k} * \mu_{\alpha} \quad(\alpha \in \mathbb{C}, k \in \mathbb{Z}) .
$$

Thus, for $r \mid n$, we also have $c^{(\alpha)}(n, r)=\phi_{1}^{(\alpha)}(r)$. Properties of the function $\phi_{k}^{(\alpha)}$ can also be found in [11].

Theorem 2.1. Assume that $h(p) \neq 0$ for all primes $p$. Let $\alpha \in \mathbb{C}$ and $n, r \in \mathbb{N}$ with prime factorizations $n=\prod p^{a}, r=\prod p^{b}$, where $a, b$ are nonnegative integers. Then

$$
f_{\alpha}(n, r)=\prod_{p}\left\{F_{\alpha}\left(p^{b}\right)-h(p)^{a+1} F_{\alpha}\left(p^{b-a-1}\right)\right\},
$$

with the convention that $F_{\alpha}\left(p^{s}\right)=0$ for negative integer $s$.

Proof. If $\operatorname{gcd}\left(n_{1}, n_{2}\right)=\operatorname{gcd}\left(r_{1}, r_{2}\right)=\operatorname{gcd}\left(n_{1}, r_{2}\right)=\operatorname{gcd}\left(n_{2}, r_{1}\right)=1$, then $\operatorname{gcd}\left(\operatorname{gcd}\left(n_{1}, r_{1}\right)\right.$, $\left.\operatorname{gcd}\left(n_{2}, r_{2}\right)\right)=1$ and $\operatorname{gcd}\left(n_{1} n_{2}, r_{1} r_{2}\right)=\operatorname{gcd}\left(n_{1}, r_{1}\right) \operatorname{gcd}\left(n_{2}, r_{2}\right)$. By multiplicativity, we have

$$
\begin{aligned}
f_{\alpha}\left(n_{1} n_{2}, r_{1} r_{2}\right) & =\sum_{d \mid \operatorname{gcd}\left(n_{1} n_{2}, r_{1} r_{2}\right)} h(d) g\left(\frac{r_{1} r_{2}}{d}\right) \mu_{\alpha}\left(\frac{r_{1} r_{2}}{d}\right) \\
& =\sum_{d_{1} \mid \operatorname{gcd}\left(n_{1}, r_{1}\right)} h\left(d_{1}\right) g\left(\frac{r_{1}}{d_{1}}\right) \mu_{\alpha}\left(\frac{r_{1}}{d_{1}}\right) \sum_{d_{2} \mid \operatorname{gcd}\left(n_{2}, r_{2}\right)} h\left(d_{2}\right) g\left(\frac{r_{2}}{d_{2}}\right) \mu_{\alpha}\left(\frac{r_{2}}{d_{2}}\right) \\
& =f_{\alpha}\left(n_{1}, r_{1}\right) f_{\alpha}\left(n_{2}, r_{2}\right) .
\end{aligned}
$$

Using (2.6), it suffices to evaluate $f_{\alpha}$ at prime powers in each of its variables.

If $b \leq a$, then $f_{\alpha}\left(p^{a}, p^{b}\right)=\sum_{d \mid p^{b}} h(d) g\left(p^{b} / d\right) \mu_{\alpha}\left(p^{b} / d\right)=F_{\alpha}\left(p^{b}\right)$.

If $b>a$, then

$$
f_{\alpha}\left(p^{a}, p^{b}\right)=\sum_{i=0}^{a} h\left(p^{i}\right) g\left(p^{b-i}\right) \mu_{\alpha}\left(p^{b-i}\right)=F_{\alpha}\left(p^{b}\right)-\sum_{i=a+1}^{b} h\left(p^{i}\right) g\left(p^{b-i}\right) \mu_{\alpha}\left(p^{b-i}\right) .
$$

Consequently,

$$
\begin{aligned}
f_{\alpha}(n, r) & \left.=\prod_{p}\left[F_{\alpha}\left(p^{b}\right)-\sum_{i=a+1}^{b} h\left(p^{i}\right) g\left(p^{b-i}\right) \mu_{\alpha}\left(p^{b-i}\right)\right] \quad \text { (where empty sum is taken to be } 0\right) \\
& =\prod_{p}\left\{F_{\alpha}\left(p^{b}\right)-h(p)^{a+1} F_{\alpha}\left(p^{b-a-1}\right)\right\} .
\end{aligned}
$$


The following corollary, known as Hölder relation, is [2, Theorem 8.8] and [15, Theorem 2.3]. It is a special case of a more general result in $[1,6]$, so we omit its proof.

Corollary 2.2. If $h(p) \neq 0$ and $h(p) \neq g(p)$ for all primes $p$, then

$$
f_{1}(n, r)=\frac{F_{1}(r) g(m) \mu(m)}{F_{1}(m)}, \quad \text { where } m=\frac{r}{\operatorname{gcd}(n, r)} \text {. }
$$

Corollary 2.3. Let $G(r):=\left(\zeta_{1} * \zeta_{0} \mu_{\alpha}\right)(r)$. Then for $n=\prod p^{a}, r=\prod p^{b}$,

$$
c^{(\alpha)}(n, r)=\prod_{p}\left\{G\left(p^{b}\right)-p^{a+1} G\left(p^{b-a-1}\right)\right\} .
$$

Proof. Put $h=\zeta_{1}, g=\zeta_{0}$ in Theorem 2.1.

Theorem 2.4. If $h(p) \neq 0$ for all primes $p$ and $H=1 / h$, then for any $n, r \in \mathbb{N}$,

$$
F_{\alpha}(r) \sum_{\substack{d \mid r \\ \operatorname{gcd}(n, d)=1}} \frac{h(d)}{F_{\alpha}(d)} \mu_{\alpha}\left(\frac{r}{d}\right)=\prod_{p \mid n}\left(F_{\alpha} \mu_{\alpha}\right)\left(p^{v_{p}(r)}\right) \prod_{p \nmid n}\left(F_{\alpha} h\left(\frac{1}{F_{\alpha}} * H^{-\alpha}\right)\right)\left(p^{v_{p}(r)}\right) .
$$

Proof. Let

$$
S(r)=F_{\alpha}(r) \sum_{\substack{d \mid r \\ \operatorname{gcd}(n, d)=1}} \frac{h(d)}{F_{\alpha}(d)} \mu_{\alpha}\left(\frac{r}{d}\right)
$$

Then $S$ is multiplicative. It suffices to consider the value of $S$ at $p^{a}$ for $p$ prime and $a \in \mathbb{N}$. If $p \mid n$, then $S\left(p^{a}\right)=F_{\alpha}\left(p^{a}\right) \mu_{\alpha}\left(p^{a}\right)$. If $p \nmid n$, then

$$
\begin{aligned}
S\left(p^{a}\right) & =F_{\alpha}\left(p^{a}\right) h(p)^{a}\left[\frac{\mu_{\alpha}\left(p^{a}\right)}{F_{\alpha}(1) h(p)^{a}}+\frac{\mu_{\alpha}\left(p^{a-1}\right)}{F_{\alpha}(p) h(p)^{a-1}}+\cdots+\frac{\mu_{\alpha}(1)}{F_{\alpha}\left(p^{a}\right) h(1)}\right] \\
& =F_{\alpha}\left(p^{a}\right) h(p)^{a}\left(\frac{1}{F_{\alpha}} * H^{-\alpha}\right)\left(p^{a}\right)
\end{aligned}
$$

because $\mu_{\alpha} H=H^{-\alpha}, H=1 / h$ being completely multiplicative.

It is of interest to see how far the nice form of Corollary 2.2 can be extended. The next theorem shows that a general result of this sort is derivable based solely upon the concept of unitary pair. We say that $\langle n, r\rangle \in \mathbb{N} \times \mathbb{N}$ is a unitary pair if $\operatorname{gcd}(\operatorname{gcd}(n, r), r / \operatorname{gcd}(n, r))=1$. Theorem 2.5. Assume that $h(p) \neq 0$ for all primes $p$. Let $\langle n, r\rangle$ be a unitary pair and $N=r / \operatorname{gcd}(n, r)$. Then

$$
f_{\alpha}(n, r)=\frac{g(N) \mu_{\alpha}(N) F_{\alpha}(r)}{F_{\alpha}(N)},
$$

whenever $F_{\alpha}(N) \neq 0$, where $F_{\alpha}(r)=\left(h * g \mu_{\alpha}\right)(r)$. 
6 Ramanujan sums via generalized Möbius functions

Proof. For any $k \in \mathbb{N}$, we see that

$$
F_{\alpha}(k)=\sum_{d \mid k} h\left(\frac{k}{d}\right) g(d) \mu_{\alpha}(d)=h(k) \sum_{d \mid k} \frac{g(d)}{h(d)} \mu_{\alpha}(d)
$$

Let $a=\operatorname{gcd}(n, r)$. Since $r=a N$ and $\langle n, r\rangle$ is a unitary pair, then $\operatorname{gcd}(a, N)=1$. Thus

$$
\begin{aligned}
f_{\alpha}(n, r) & =\sum_{d \mid a} h\left(\frac{a}{d}\right) g(N d) \mu_{\alpha}(N d)=g(N) \mu_{\alpha}(N) h(a) \sum_{d \mid a} \frac{g(d) \mu_{\alpha}(d)}{h(d)} \\
& =g(N) \mu_{\alpha}(N) F_{\alpha}(a) \frac{F_{\alpha}(N)}{F_{\alpha}(N)}=g(N) \mu_{\alpha}(N) \frac{F_{\alpha}(r)}{F_{\alpha}(N)} .
\end{aligned}
$$

Corollary 2.6. Let $\langle n, r\rangle$ be a unitary pair and $N=r / \operatorname{gcd}(n, r)$. Then

$$
c^{(\alpha)}(n, r)=\frac{\mu_{\alpha}(N) \phi_{1}^{(\alpha)}(r)}{\phi_{1}^{(\alpha)}(N)}
$$

whenever $\phi_{1}^{(\alpha)}(N) \neq 0$.

Proof. Take $h=\zeta_{1}, g=\zeta_{0}$ in Theorem 2.5.

The next lot of identities relates to the Cauchy product of GRSs. In the classical situation, these identities are used in the representation of even functions with respect to the usual Ramanujan sums.

Theorem 2.7. Let $\alpha, \beta \in \mathbb{C}$ and $n, r \in \mathbb{N}$. If $r$ is divisible by both $s$ and $t$, then

$$
\sum_{a+b \equiv n(\bmod r)} c^{(\alpha)}(a, s) c^{(\beta)}(b, t)=r \sum_{k=0}^{q-1} \mu_{\alpha-1}\left(s_{1} \operatorname{gcd}(k, g)\right) \mu_{\beta-1}\left(t_{1} \operatorname{gcd}(k, g)\right) e^{2 \pi i k n / g}
$$

where $g=\operatorname{gcd}(s, t), s=g s_{1}, t=g t_{1}, \operatorname{gcd}\left(s_{1}, t_{1}\right)=1$.

Proof. To prove Theorem 2.7, we make use of the following auxiliary result:

$$
\sum_{a+b \equiv n(\bmod r)} e^{2 \pi i a s / r} e^{2 \pi i b t / r}= \begin{cases}r e^{2 \pi i t r(a+b) / r}=r e^{2 \pi i t n / r}, & \text { if } \frac{s-t}{r} \in \mathbb{Z}, \\ 0, & \text { otherwise. }\end{cases}
$$

Since

$$
\begin{aligned}
& \sum_{a+b \equiv n(\bmod r)} c^{(\alpha)}(a, s) c^{(\beta)}(b, t) \\
& \quad=\sum_{m(\bmod s)} \sum_{j(\bmod t)} \mu_{\alpha-1}(\operatorname{gcd}(m, s)) \mu_{\beta-1}(\operatorname{gcd}(j, t)) \sum_{a+b \equiv n(\bmod r)} e^{2 \pi i(m a / s+j b / t)},
\end{aligned}
$$


putting $s_{1}=r / s$ and $t_{1}=r / t$, we get

$$
\begin{aligned}
& \sum_{a+b=n(\bmod r)} c^{(\alpha)}(a, s) c^{(\beta)}(b, t) \\
& = \begin{cases}\sum_{m(\bmod s)} \sum_{j(\bmod t)} \mu_{\alpha-1}(\operatorname{gcd}(m, s)) \mu_{\beta-1}(\operatorname{gcd}(j, t)) r e^{2 \pi i m s_{1} n / r}, & \text { if } \frac{m s_{1}-j t_{1}}{r} \in \mathbb{Z}, \\
0, & \text { otherwise }\end{cases} \\
& = \begin{cases}\sum_{m(\bmod s)} \sum_{j(\bmod t)} \mu_{\alpha-1}(\operatorname{gcd}(m, s)) \mu_{\beta-1}(\operatorname{gcd}(j, t)) r e^{2 \pi i m n / s}, & \text { if } \frac{m}{s}-\frac{j}{t} \in \mathbb{Z}, \\
0, & \text { otherwise }\end{cases} \\
& =r \sum_{\substack{m(\bmod s) \\
m t=0(\bmod s)}} \mu_{\alpha-1}(\operatorname{gcd}(m, s)) \mu_{\beta-1}\left(\operatorname{gcd}\left(\frac{m t}{s}, t\right)\right) e^{2 \pi i m n / s}, \\
& =
\end{aligned}
$$

where $g=\operatorname{gcd}(s, t), s=g s_{1}, t=g t_{1}, \operatorname{gcd}\left(s_{1}, t_{1}\right)=1$.

A special case of Theorem 2.7, which is [15, Theorem 2.6], yields an orthogonality property which is used in the classical representation problem.

Corollary 2.8. If $r$ is divisible by both $s$ and $t$, then

$$
\sum_{a+b \equiv n(\bmod r)} c(a, s) c(b, t)= \begin{cases}r c(n, s), & \text { if } s=t, \\ 0, & \text { otherwise. }\end{cases}
$$

Proof. Taking $\alpha=\beta=1$ in Theorem 2.7, we have

$$
\sum_{a+b \equiv n(\bmod r)} c(a, s) c(b, t)=r \sum_{k=0}^{g-1} \mu_{0}\left(s_{1} \operatorname{gcd}(k, g)\right) \mu_{0}\left(t_{1} \operatorname{gcd}(k, g)\right) e^{2 \pi i k n / g},
$$

where $g=\operatorname{gcd}(s, t), s=g s_{1}, t=g t_{1}, \operatorname{gcd}\left(s_{1}, t_{1}\right)=1$. Since $s=t \Leftrightarrow g=s=t, s_{1}=1, t_{1}=1$, we deduce

$$
\sum_{a+b \equiv n(\bmod r)} c(a, s) c(b, t)= \begin{cases}r \sum_{\substack{k=0 \\ \operatorname{gcd}(k, s)=1}}^{s-1} e^{2 \pi i k n / s}=r c(n, s), & \text { if } s=t, \\ 0, & \text { otherwise. }\end{cases}
$$


8 Ramanujan sums via generalized Möbius functions

Our last batch of identities deals with the Dirichlet series of GRSs. Recall from [22] that the Dirichlet series associated with each GRS, which converges for $\operatorname{Re} s>1$, is

$$
\sum_{n=1}^{\infty} \frac{\mu_{\alpha}(n)}{n^{s}}=\frac{1}{\zeta^{\alpha}(s)}=\left(\sum_{n=1}^{\infty} \frac{1}{n^{s}}\right)^{-\alpha}
$$

We now use a technique of Ramanathan [18] to derive extensions of this result. For a multiplicative function $f$, define $F_{m}(n)=f(m n) / f(m)(n, m \in \mathbb{N})$. It is proved in [18] that

$$
\sum_{n=1}^{\infty} \frac{F_{m}(n)}{n^{s}}=\sum_{n=1}^{\infty} \frac{f(n)}{n^{s}} \prod_{p \mid m} \mathscr{G}(p, s)
$$

where

$$
\mathscr{G}(p, s):=\frac{G_{v_{p}(m)}(p, s)}{G_{0}(p, s)}, \quad G_{k}(p, s):=1+\frac{1}{f\left(p^{k}\right)} \sum_{i \geq 1} \frac{f\left(p^{k+i}\right)}{p^{i s}} .
$$

The finite-product term simplifies radically in two special cases. If $f$ is a specially multiplicative function [13], that is, a Dirichlet product of two completely multiplicative functions, then

$$
\mathscr{G}(p, s)=1-\frac{B(p) f\left(p^{v_{p}(M)-1}\right)}{f\left(p^{v_{p}(M)}\right) p^{s}}, \quad B(p):=(f(p))^{2}-f\left(p^{2}\right) .
$$

If $f$ is a totient [13], that is, a Dirichlet product of a completely multiplicative function with an inverse of a completely multiplicative function, then

$$
G(p, s)=\frac{1}{1-G(p) p^{-s}}, \quad G(p):=\frac{f\left(p^{2}\right)-(f(p))^{2}}{f(p)} .
$$

Taking $f=\mu_{\alpha}$, we have the following.

Theorem 2.9. If $\alpha \in \mathbb{C}$ and $m, s \in \mathbb{N}$, then

$$
\sum_{n=1}^{\infty} \frac{\mu_{\alpha}(m n)}{n^{s}}=\frac{\mu_{\alpha}(m)}{\zeta^{\alpha}(s)} \prod_{p \mid m} \frac{1+\sum_{i \geq 1}\left(\mu_{\alpha}\left(p^{v_{p}(m)+i}\right) / \mu_{\alpha}\left(p^{v_{p}(m)}\right) p^{i s}\right)}{1+\sum_{i \geq 1}\left(\mu_{\alpha}\left(p^{i}\right) / p^{i s}\right)} .
$$

Putting $\alpha=1$ in Theorem 2.9, we get yet another beautiful identity

$$
\sum_{n=1}^{\infty} \frac{\mu(m n)}{n^{s}}=\frac{\mu(m)}{\zeta(s)} \prod_{p \mid m} \frac{1}{1-p^{-s}}
$$




\section{Representation of even functions}

For a fixed $r \in \mathbb{N}$, an arithmetic function $f$ is called an even function $(\bmod r)$ if $f(\operatorname{gcd}(n, r))=f(n)$ for all $n \in \mathbb{N}$. It is well known [15, Theorem 2.9] that an even function $(\bmod r), f$, is uniquely representable in the form

$$
f(n)=\sum_{d \mid r} a(d) c(n, d) \quad(n \in \mathbb{N}),
$$

where the Fourier coefficients $a(d)$ are given by

$$
a(d)=\frac{1}{r} \sum_{e \mid r} f\left(\frac{r}{e}\right) c\left(\frac{r}{d}, e\right)=\frac{1}{r \phi(d)} \sum_{m=1}^{r} f(m) c(m, d) .
$$

As expected, the GRS is an even function $(\bmod r)$ as recorded in the next lemma whose straight-forward proof is omitted.

Lemma 3.1. For a fixed $r \in \mathbb{N}, \alpha \in \mathbb{C}$, the $G R S c^{(\alpha)}(n, r)$ is an even function $(\bmod r)$ of $n$.

Using [15, Theorem 2.9], we have the following.

Proposition 3.2. For fixed $\alpha \in \mathbb{C}, r \in \mathbb{N}$, the $G R S c^{(\alpha)}(n, r)$ is uniquely representable in the form

$$
c^{(\alpha)}(n, r)=\sum_{d \mid r} a_{r}^{(\alpha)}(d) c(n, d) \quad(n \in \mathbb{N})
$$

where the coefficients $a_{r}^{(\alpha)}(d), d \mid r$, are given by

$$
a_{r}^{(\alpha)}(d)=\frac{1}{r} \sum_{e \mid r} c^{(\alpha)}\left(\frac{r}{e}, r\right) c\left(\frac{r}{d}, e\right)=\frac{1}{r \phi(d)} \sum_{m=1}^{r} c^{(\alpha)}(m, r) c(m, d) .
$$

Using identities established in the last section, we record the following identities involving the coefficients $a_{r}^{(\alpha)}(d)$. This is a special case of a more general result in $[4,10]$, so we omit its proof.

Theorem 3.3. Let $\alpha, \beta \in \mathbb{C} ; n, r, s, t \in \mathbb{N}$ with $r$ divisible by $s$ and $t$. Then

$$
\sum_{a+b \equiv n(\bmod r)} c^{(\alpha)}(a, s) c^{(\beta)}(b, t)=\sum_{s_{1} \mid \operatorname{gcd}(s, t)} a_{s}^{(\alpha)}\left(s_{1}\right) a_{t}^{(\beta)}\left(s_{1}\right) r c\left(n, s_{1}\right),
$$

where the coefficients $a_{s}^{(\alpha)}(\cdot), a_{t}^{(\beta)}(\cdot)$ are as in Proposition 3.2.

Two remarks are now in order. First, recall from [15, Theorem 2.8] that for $r, e_{1}, e_{2} \in \mathbb{N}$ with $r$ divisible by both $e_{1}$ and $e_{2}$, we have

$$
\sum_{d \mid r} c\left(\frac{r}{d}, e_{1}\right) c\left(\frac{r}{e_{2}}, d\right)= \begin{cases}r, & \text { if } e_{1}=e_{2} \\ 0, & \text { otherwise }\end{cases}
$$


Incorporating with Proposition 3.2, we get

$$
a_{r}^{(1)}(d)=\frac{1}{r} \sum_{t \mid r} c\left(\frac{r}{t}, r\right) c\left(\frac{r}{d}, t\right)= \begin{cases}\frac{1}{r} r=1, & \text { if } r=d \\ 0, & \text { otherwise. }\end{cases}
$$

Second, taking $\alpha=\beta=1$ and $s, t \mid r$ in Theorem 3.3 and using (3.7), we have

$$
\sum_{a+b \equiv n(\bmod r)} c(a, s) c(b, t)=\sum_{s_{1} \mid \operatorname{gcd}(s, t)} a_{s}^{(1)}\left(s_{1}\right) a_{t}^{(1)}\left(s_{1}\right) r c\left(n, s_{1}\right)= \begin{cases}r c(n, s), & \text { if } s=t, \\ 0, & \text { otherwise }\end{cases}
$$

which is [15, Theorem 2.6].

Though Theorem 3.3 does not provide us with any obvious orthogonality relation between two GRSs, we may be able to salvage such relation in certain special cases.

Theorem 3.4. Let $\alpha \in \mathbb{C}$ and $r, s, t \in \mathbb{N}$ with $r$ divisible by both $s$ and $t$. Then

$$
\sum_{d \mid r} c^{(\alpha)}\left(\frac{r}{d}, s\right) c\left(\frac{r}{t}, d\right)= \begin{cases}a_{s}^{(\alpha)}(t) r, & \text { if } t \mid s \\ 0, & \text { otherwise. }\end{cases}
$$

Proof. By Proposition 3.2 and (3.6),

$$
\begin{aligned}
\sum_{d \mid r} c^{(\alpha)}\left(\frac{r}{d}, s\right) c\left(\frac{r}{t}, d\right) & =\sum_{x \mid s} a_{s}^{(\alpha)}(x) \sum_{d \mid r} c\left(\frac{r}{d}, x\right) c\left(\frac{r}{t}, d\right) \\
& =\sum_{x \mid s} a_{s}^{(\alpha)}(x) \begin{cases}r, & \text { if } x=t, \\
0, & \text { otherwise }\end{cases} \\
& = \begin{cases}a_{s}^{(\alpha)}(t) r, & \text { if } t \mid s, \\
0, & \text { otherwise. }\end{cases}
\end{aligned}
$$

Theorem 3.5. Let $\alpha, \beta \in \mathbb{C}, r, s, t \in \mathbb{N}$ with $r$ divisible by both $s$ and $t$. Then

$$
\sum_{d \mid r} c^{(\alpha)}\left(\frac{r}{d}, s\right) c^{(\beta)}\left(\frac{r}{d}, t\right) \phi(d)=\sum_{a+b \equiv 0(\bmod r)} c^{(\alpha)}(a, s) c^{(\beta)}(b, t) .
$$

Proof. Observe that

$$
\begin{aligned}
\sum_{a+b \equiv 0(\bmod r)} c^{(\alpha)}(a, s) c^{(\beta)}(b, t) & =\sum_{a=1}^{r} c^{(\alpha)}(a, s) c^{(\beta)}(-a, t) \\
& =\sum_{d \mid r} \sum_{\substack{1 \leq x \leq d \\
\operatorname{gcd}(x, d)=1}} c^{(\alpha)}\left(\frac{x r}{d}, s\right) c^{(\beta)}\left(\frac{-x r}{d}, t\right) \\
& =\sum_{d \mid r} \sum_{x} c^{(\alpha)}\left(\frac{x r}{d}, s\right) c^{(\beta)}\left(\frac{-x r}{d}, t\right)
\end{aligned}
$$


where the last sum over $x$ runs over any reduced residues system $(\bmod d)$. Furthermore, we can assume that this reduced residue system $(\bmod d)$ is contained in a reduced residue system $(\bmod r)$, that is, that $\operatorname{gcd}(x, r)=1$ for each $x$. Thus $c^{(\alpha)}(x r / d, s)=$ $c_{s}^{(\alpha)}(\operatorname{gcd}(x r / d, s), s)$ and $c^{(\beta)}(-x r / d, t)=c_{t}^{(\beta)}(\operatorname{gcd}(-x r / d, t), t)$. Since $\operatorname{gcd}(x, r)=\operatorname{gcd}(x, s)=$ $\operatorname{gcd}(x, t)=1$, we have $c^{(\alpha)}(x r / d, s)=c^{(\alpha)}(r / d, s), c^{(\beta)}(-x r / d, t)=c^{(\beta)}(r / d, t)$ and the result follows.

There is an alternative proof of Theorem 3.5, using Proposition 3.2 [15, Problem 2.20 on page 93], (3.6), and Theorem 3.3 which proceeds as follows:

$$
\begin{aligned}
\sum_{d \mid r} c^{(\alpha)}\left(\frac{r}{d}, s\right) c^{(\beta)}\left(\frac{r}{d}, t\right) \phi(d) & =\sum_{d \mid r} \sum_{d_{1} \mid s} \sum_{d_{2} \mid t} a_{s}^{(\alpha)}\left(d_{1}\right) a_{t}^{(\beta)}\left(d_{2}\right) \phi(d) c\left(\frac{r}{d}, d_{1}\right) c\left(\frac{r}{d}, d_{2}\right) \\
& =\sum_{d_{1} \mid s} \sum_{d_{2} \mid t} a_{s}^{(\alpha)}\left(d_{1}\right) a_{t}^{(\beta)}\left(d_{2}\right) \sum_{d \mid r} c\left(\frac{r}{d}, d_{1}\right) \phi\left(d_{2}\right) c\left(\frac{r}{d_{2}}, d\right) \\
& =\sum_{d \mid \operatorname{gcd}(s, t)} a_{s}^{(\alpha)}(d) a_{t}^{(\beta)}(d) \phi(d) r=\sum_{d \mid \operatorname{gcd}(s, t)} a_{s}^{(\alpha)}(d) a_{t}^{(\beta)}(d) r c(0, d) \\
& =\sum_{a+b \equiv 0(\bmod r)} c^{(\alpha)}(a, s) c^{(\beta)}(b, t) .
\end{aligned}
$$

The next result provides a main tool for our representation problem.

Lemma 3.6. For a fixed $\alpha \in \mathbb{C}, a_{r}^{(\alpha)}(r)$ is a multiplicative function of $r \in \mathbb{N}$.

Proof. Let $r_{1}, r_{2} \in \mathbb{N}$ be such that $\operatorname{gcd}\left(r_{1}, r_{2}\right)=1$. Then

$$
\begin{aligned}
a_{r_{1} r_{2}}^{(\alpha)}\left(r_{1} r_{2}\right) & =\frac{1}{r_{1} r_{2}} \sum_{\substack{e_{1}\left|r_{1} \\
e_{2}\right| r_{2}}} c^{(\alpha)}\left(\frac{r_{1}}{e_{1}} \cdot \frac{r_{2}}{e_{2}}, r_{1} r_{2}\right) \mu\left(e_{1} e_{2}\right) \\
& =\frac{1}{r_{1} r_{2}} \sum_{\substack{e_{1}\left|r_{1} \\
e_{2}\right| r_{2}}} c^{(\alpha)}\left(\frac{r_{1}}{e_{1}}, r_{1}\right) c^{(\alpha)}\left(\frac{r_{2}}{e_{2}}, r_{2}\right) \mu\left(e_{1}\right) \mu\left(e_{2}\right) \\
& =a_{r_{1}}^{(\alpha)}\left(r_{1}\right) a_{r_{2}}^{(\alpha)}\left(r_{2}\right) .
\end{aligned}
$$

Theorem 3.7. For each $n \in \mathbb{N}$ and $\alpha \in \mathbb{C}$, then $a_{n}^{(\alpha)}(n)=1$.

Proof. By Lemma 3.6 and the fact that $a_{1}^{(\alpha)}(1)=1$, it suffices to check that $a_{p^{k}}^{(\alpha)}\left(p^{k}\right)=1$ for each prime $p$ and each $k \in \mathbb{N}$. This follows at once by direct computation.

It is worthwhile to carry out some explicit computations of the coefficient values. Of interest is another eye-catching formula. 
Proposition 3.8. For $r \in \mathbb{N}$, then $a_{r}^{(\alpha)}(1)=\mu_{\alpha-1}(r)$.

Proof. This follows easily from

$$
a_{r}^{(\alpha)}(1)=\frac{1}{r} \sum_{m=1}^{r} \sum_{d \mid g c d(m, r)} d \mu_{\alpha}\left(\frac{r}{d}\right)=\frac{1}{r} \sum_{d \mid r} d \mu_{\alpha}\left(\frac{r}{d}\right) \cdot \frac{r}{d}=\left(\mu_{\alpha} * u\right)(r)=\mu_{\alpha-1}(r) .
$$

We come now to the main point of this section. It is natural to ask whether the set of GRSs can be used as a basis for Fourier expansions just as the classical Ramanujan sums can. We will show that the answer is affirmative. To do so, it suffices to show that Ramanujan sums can be written as a linear combination of GRSs, that is, the coefficients $A(t ; D)$ with $D \mid t$ can be so determined that

$$
c(n, t)=\sum_{D \mid t} A(t ; D) c^{(\alpha)}(n, D)
$$

Using the unique expansion of GRSs with respect to Ramanujan sums, this is equivalent to finding $A(t ; D)$ so that

$$
c(n, t)=\sum_{D \mid t} A(t ; D) \sum_{d \mid D} a_{D}^{(\alpha)}(d) c(n, d)=\sum_{d \mid t} c(n, d) \sum_{k \mid(t / d)} A(t ; k d) a_{k d}^{(\alpha)}(d) .
$$

It thus suffices to show that the condition

$$
\sum_{k \mid t / d} A(t ; k d) a_{k d}^{(\alpha)}(d)= \begin{cases}0, & \text { if } d \neq t, d \mid t \\ 1, & \text { if } d=t\end{cases}
$$

well defines the $A(t ; D)$ 's. For $d=t$, condition $(\mathrm{R})$ requires that

$$
1=\sum_{k \mid 1} A(d ; k d) a_{k d}^{(\alpha)}(d)=A(t ; t) a_{t}^{(\alpha)}(t)=A(t ; t),
$$

the last equality being followed from Theorem 3.7, which uniquely determines $A(t, t)$. For $t=2 d$, condition $(\mathrm{R})$ requires that

$$
0=\sum_{k \mid 2} A(2 d ; k d) a_{k d}^{(\alpha)}(d)=A(2 d ; d) a_{d}^{(\alpha)}(d)+A(2 d ; 2 d) a_{2 d}^{(\alpha)}(d)=A(2 d ; d)+a_{2 d}^{(\alpha)}(d) .
$$

Using Theorem 3.7 and the previous case, we have $A(2 d ; d)=-a_{2 d}^{(\alpha)}(d)$. In general, for $n, k \in \mathbb{N}$, assuming $A(n d ; k d)$ have already been well determined for $k \mid n$ and $k>1$ but not for $k=1$, condition $(\mathrm{R})$ requires that

$$
0=\sum_{k \mid n} A(n d ; k d) a_{k d}^{(\alpha)}(d)=A(n d ; d) a_{d}^{(\alpha)}(d)+\sum_{k \mid n, k>1} A(n d ; k d) a_{k d}^{(\alpha)}(d)
$$


and so

$$
A(n d ; d)=-\sum_{k \mid n, k>1} A(n d ; k d) a_{k d}^{(\alpha)}(d) .
$$

Using the induction hypothesis and Theorem 3.7, we see at once that $A(n d ; d)$ is well determined. Consequently, any even function $(\bmod r)$ can be expanded as a Fourier expansion with respect to the GRSs.

To prove the uniqueness of such expansion, it suffices to show that the zero function, which is clearly even $(\bmod r)$, has only zero Fourier coefficients. Writing the expansion of the zero function with respect to the GRSs and using Proposition 3.2, we get

$$
0=\sum_{D \mid r} B(D) c^{(\alpha)}(n, D)=\sum_{D \mid r} B(D) \sum_{m \mid D} a_{D}^{(\alpha)}(m) c(n, m)=\sum_{m \mid r} c(n, m) \sum_{k \mid r / m} B(k m) a_{k m}^{(\alpha)}(m) .
$$

By the uniqueness of the expansion with respect to the usual Ramanujan sums, we deduce

$$
\sum_{k \mid r / m} B(k m) a_{k m}^{(\alpha)}(m)=0 .
$$

Let the prime factorization of $r$ be $r=p_{1}^{n_{1}} p_{2}^{n_{2}} \cdots p_{s}^{n_{s}}$. Putting $m=r$, we get

$$
0=\sum_{k \mid 1} B(k r) a_{k r}^{(\alpha)}(r)=B(r) a_{r}^{(\alpha)}(r)=B(r)=B\left(p_{1}^{n_{1}} p_{2}^{n_{2}} \cdots p_{s}^{n_{s}}\right)
$$

Taking $m=p_{1}^{n_{1}} p_{2}^{n_{2}} \cdots p_{s}^{n_{s}-1}$ and using the result just found, we get

$$
0=\sum_{k \mid p_{s}} B(k m) a_{k m}^{(\alpha)}(m)=B(m) a_{m}^{(\alpha)}(m)+B(r) a_{r}^{(\alpha)}(m)=B(m)=B\left(p_{1}^{n_{1}} p_{2}^{n_{2}} \cdots p_{s}^{n_{s}-1}\right) .
$$

In general, by reverse induction on the number of prime divisors of $r$, counted with multiplicity, we obtain $B(m)=0$ for $m \mid r$. Summarizing, we have the following.

THeOREM 3.9. An even function $(\bmod r) f$ can be uniquely expanded as a Fourier expansion in the form

$$
f(n)=\sum_{D \mid r} A^{(\alpha)}(D) c^{(\alpha)}(n, D)
$$

where the Fourier coefficients $A^{(\alpha)}(D)$ are given by

$$
A^{(\alpha)}(D)=\sum_{j \mid r / D} a(j D) A(j D ; D),
$$

with

$$
a(d)=\frac{1}{r} \sum_{e \mid r} f\left(\frac{r}{e}\right) c\left(\frac{r}{d}, e\right)=\frac{1}{r \phi(d)} \sum_{m=1}^{r} f(m) c(m, d),
$$


14 Ramanujan sums via generalized Möbius functions

and the $A(j D ; D)$ are uniquely determined from

$$
\sum_{k \mid t / d} A(t ; k d) a_{k d}^{(\alpha)}(d)= \begin{cases}0, & \text { if } d \neq t, d \mid t, \\ 1, & \text { if } d=t\end{cases}
$$

with $a_{k d}^{(\alpha)}(d)$ as defined in Proposition 3.2.

\section{Mean value}

The mean value of an arithmetic function $f$ is defined (see $[5,19,23])$ by

$$
M(f):=\lim _{x \rightarrow \infty} \frac{1}{x} \sum_{n \leq x} f(n),
$$

whenever this limit exists. For a GRS, its mean value is quite simple.

Theorem 4.1. For a fixed $r \in \mathbb{N}$ and $\alpha \in \mathbb{C}$, then $M\left(c^{(\alpha)}(\cdot, r)\right)=\mu_{\alpha-1}(r)$.

Proof. Since

$$
\begin{aligned}
\sum_{n \leq x} c^{(\alpha)}(n, r) & =\sum_{n \leq x} \sum_{d \mid \operatorname{gcd}(n, r)} d \mu_{\alpha}\left(\frac{r}{d}\right) \\
& =\sum_{d \mid r} d \mu_{\alpha}\left(\frac{r}{d}\right) \sum_{\substack{n \leq x \\
d \mid n}} 1=x \sum_{d \mid r} \mu_{\alpha}\left(\frac{r}{d}\right)-\sum_{d \mid r} d \mu_{\alpha}\left(\frac{r}{d}\right)\left(\frac{x}{d}-\left[\frac{x}{d}\right]\right) \\
& =x\left(\mu_{\alpha} * u\right)(r)+R_{r}(x)=x \mu_{\alpha-1}(r)+R_{r}(x)
\end{aligned}
$$

where $R_{r}(x)=\sum_{d \mid r} d \mu_{\alpha}(r / d)(x / d-[x / d])$, the result follows at once from the fact that $R_{r}(x)=o(x)$.

Invoking upon [23, Proposition 1], Theorem 4.1 yields.

Corollary 4.2. Let $r \in \mathbb{N}$ and $\alpha \in \mathbb{C}$. Then

$$
\mu_{\alpha-1}(r)=M\left(c^{(\alpha)}(\cdot, r)\right)=\frac{1}{r} \sum_{e \mid r} c^{(\alpha)}(e, r) \phi\left(\frac{r}{e}\right) .
$$

In order to evaluate the mean value of the product of two GRSs, we use the next lemma whose straightforward proof is omitted.

Lemma 4.3. If $f, g$ are arithmetic functions and $\beta \in \mathbb{C}$, then

$$
M(f+g)=M(f)+M(g), \quad M(\beta f)=\beta M(f) .
$$

Theorem 4.4. If $r, s \in \mathbb{N}$ and $\alpha \in \mathbb{C}$, then

$$
M\left(c^{(\alpha)}(\cdot, r) c^{(\alpha)}(\cdot, s)\right)=\sum_{d \mid(r, s)} a_{r}^{(\alpha)}(d) a_{s}^{(\alpha)}(d) \phi(d) .
$$


Proof. Using the orthogonality relation of the usual Ramanujan sums as in [23, equation (2)] together with Lemma 4.3, we get

$$
\begin{aligned}
M\left(c^{(\alpha)}(\cdot, r) c^{(\alpha)}(\cdot, s)\right) & =\sum_{d \mid r} \sum_{e \mid s} a_{r}^{(\alpha)}(d) a_{s}^{(\alpha)}(e) M(c(\cdot, d) c(\cdot, e)) \\
& =\sum_{d \mid r} \sum_{e \mid s} a_{r}^{(\alpha)}(d) a_{s}^{(\alpha)}(e) \delta_{d, e} \phi(d) \\
& =\sum_{d \mid \operatorname{gcd}(r, s)} a_{r}^{(\alpha)}(d) a_{s}^{(\alpha)}(d) \phi(d)
\end{aligned}
$$

The next corollary is immediate from Theorem 4.4 and Proposition 3.8. It reveals why an appropriate orthogonality relation between two GRSs only exists when $\alpha=1$.

Corollary 4.5. If $r, s \in \mathbb{N}$ and $\alpha \in \mathbb{C}$, then

$$
\begin{array}{r}
M\left(c^{(\alpha)}(\cdot, r) c^{(\alpha)}(\cdot, s)\right)=\mu_{\alpha-1}(r s) \quad \text { if } \operatorname{gcd}(r, s)=1, \\
M\left(c^{(\alpha)}(\cdot, r) c^{(\alpha)}(\cdot, r)\right)=\sum_{d \mid r} a_{r}^{(\alpha)}(d) a_{r}^{(\alpha)}(d) \phi(d) .
\end{array}
$$

Taking $\alpha=1$ in Theorem 4.4, using the remarks preceding Theorem 3.4, we have

$$
\begin{aligned}
M(c(\cdot, r) c(\cdot, s)) & = \begin{cases}\phi(r), & \text { if } r=s \\
0, & \text { otherwise }\end{cases} \\
& =\delta_{r, s} \phi(r),
\end{aligned}
$$

which is [23, Proposition 3].

\section{Ramanujan identities}

In this section, the remarkable identity connecting the usual Ramanujan sums with divisor functions, namely,

$$
\sigma_{s}(n)=n^{s} \zeta(s+1) \sum_{m=1}^{\infty} \frac{c(n, m)}{m^{s+1}}, \quad \text { where } \sigma_{s}(n):=\sum_{d \mid n} d^{s}=\left(\zeta_{s} * \mu_{-1}\right)(n)
$$

is extended to GRSs. Indeed, there are at least two possible extensions for this famous Ramanujan identity, which are established in the next two theorems. Our treatment for the first theorem proceeds along the line analogous to that in [21, Section 18.1]. For $\alpha \in \mathbb{C}$ and $k \in \mathbb{Z}$, define the generalized divisor function as

$$
\sigma_{k}^{(\alpha)}:=\zeta_{k} * \mu_{-\alpha}
$$

Note that $\sigma_{k}^{(\alpha)}=\phi_{k}^{(-\alpha)}$. 
16 Ramanujan sums via generalized Möbius functions

Theorem 5.1. If $n, s \in \mathbb{N}, \alpha \in \mathbb{C}$, then

$$
\frac{1}{n^{s+1}} \sum_{d \mid n} d^{s+1} \sigma_{1}^{(1-\alpha)}\left(\frac{n}{d}\right)=\sum_{k=1}^{\infty} c(n, k) \sum_{x=1}^{[t / k]} \frac{c^{(\alpha-1)}(n, k x)}{(k x)^{s+2}}
$$

for any real number $t \geq n$.

Proof. Let $\mathscr{F}(u, v)$ be a function of two variables $u$ and $v$. Define

$$
D(n)=\sum_{d \mid n} \mathscr{F}\left(d, \frac{n}{d}\right)
$$

If

$$
\eta(n, r)= \begin{cases}r, & \text { if } r \mid n, \\ 0, & \text { otherwise }\end{cases}
$$

then $D(n)$ can be written as

$$
D(n)=\sum_{x=1}^{[t]} \frac{1}{x} \eta(n, x) \mathscr{F}\left(x, \frac{n}{x}\right)
$$

where $t \geq n$. As $\sum_{d \mid r} c(n, d)=\eta(n, r)$, we have, after rewriting the last relation,

$$
D(n)=c(n, 1) \sum_{x=1}^{[t]} \frac{1}{x} \mathscr{F}\left(x, \frac{n}{x}\right)+c(n, 2) \sum_{x=1}^{[t / 2]} \frac{1}{2 x} \mathscr{F}\left(2 x, \frac{n}{2 x}\right)+\cdots
$$

Let $F(u, v)$ be a function of two variables $u$ and $v$ and take

$$
\mathscr{F}\left(d, \frac{n}{d}\right)=\frac{c^{(\alpha-1)}(n, d)}{d} F\left(d, \frac{n}{d}\right)
$$

Then

$$
D(n)=c(n, 1) \sum_{x=1}^{[t]} \frac{1}{x} \frac{c^{(\alpha-1)}(n, x)}{x} F\left(x, \frac{n}{x}\right)+c(n, 2) \sum_{x=1}^{[t / 2]} \frac{1}{2 x} \frac{c^{(\alpha-1)}(n, 2 x)}{2 x} F\left(2 x, \frac{n}{2 x}\right)+\cdots
$$

Taking $F(u, v)=v^{s}$, we get

$$
D(n)=n^{s} \sum_{k=1}^{\infty} c(n, k) \sum_{x=1}^{[t / k]} \frac{c^{(\alpha-1)}(n, k x)}{(k x)^{s+2}}
$$


On the other hand,

$$
\begin{aligned}
D(n) & =\frac{1}{n} \sum_{d \mid n} c^{(\alpha-1)}\left(n, \frac{n}{d}\right) d^{s+1}=\frac{1}{n} \sum_{d \mid n} \sum_{k \mid \operatorname{gcd}(n, n / d)} k \mu_{\alpha-1}\left(\frac{n / d}{k}\right) d^{s+1} \\
& =\frac{1}{n} \sum_{d \mid n} \sum_{k \mid n / d} k \mu_{\alpha-1}\left(\frac{n / d}{k}\right) d^{s+1}=\frac{1}{n} \sum_{d \mid n} d^{s+1}\left(\zeta_{1} * \mu_{\alpha-1}\right)\left(\frac{n}{d}\right)=\frac{1}{n} \sum_{d \mid n} d^{s+1} \sigma_{1}^{(1-\alpha)}\left(\frac{n}{d}\right),
\end{aligned}
$$

and the desired result follows.

The second possible extension looks much simpler than the first, but only holds for $\alpha$ being a positive integer.

Theorem 5.2. For a real $s>1, n \in \mathbb{N}$, if $\alpha$ is a positive integer, then

$$
(\zeta(s))^{\alpha} \sum_{r=1}^{\infty} \frac{c^{(\alpha)}(n, r)}{r^{s}}=\frac{\sigma_{s-1}(n)}{n^{s-1}} .
$$

Proof. Multiplying Dirichlet series and using Lemma 1.2 (1), we get

$$
\zeta(s) \sum_{r=1}^{\infty} \frac{c^{(\alpha)}(n, r)}{r^{s}}=\sum_{r=1}^{\infty} \frac{\left(\zeta_{0} * c^{(\alpha)}(n, \cdot)\right)}{r^{s}}(r)=\sum_{r=1}^{\infty} \frac{1}{r^{s}} \sum_{d \mid r} c^{(\alpha)}(n, d)=\sum_{r=1}^{\infty} \frac{c^{(\alpha-1)}(n, r)}{r^{s}} .
$$

Putting $S_{\alpha}=S_{\alpha}(n):=\sum_{r=1}^{\infty} c^{(\alpha)}(n, r) / r^{s}$, we find successively

$$
\zeta^{2} S_{\alpha}=\zeta S_{\alpha-1}=S_{\alpha-2}, \quad \zeta^{3} S_{\alpha}=\zeta^{2} S_{\alpha-1}=\zeta S_{\alpha-2}=S_{\alpha-3}, \ldots
$$

Thus

$$
\zeta^{\alpha} S_{\alpha}=S_{0}:=\sum_{r=1}^{\infty} \frac{c^{(0)}(n, r)}{r^{s}}=\sum_{r \mid n} \frac{1}{r^{s-1}}=\frac{\sigma_{s-1}(n)}{n^{s-1}}
$$

Theorem 5.2 can be further extended using the technique and results of Ramanathan [18], mentioned in Section 2 prior to Theorem 2.9, which readily yields the following.

Theorem 5.3. Let $\alpha, m, n \in \mathbb{N}$. If $s$ is a real number $>1$, then

$$
\sum_{k=1}^{\infty} \frac{c^{(\alpha)}(n, m k)}{k^{s}}=\frac{c^{(\alpha)}(n, m) \sigma_{s-1}(n)}{\zeta^{\alpha}(s) n^{s-1}} \prod_{p \mid m} \frac{1+\sum_{i \geq 1}\left(c^{(\alpha)}\left(n, p^{v_{p}(m)+i}\right) / c^{(\alpha)}\left(n, p^{v_{p}(m)}\right) p^{i s}\right)}{1+\sum_{i \geq 1}\left(c^{(\alpha)}\left(n, p^{i}\right) / p^{i s}\right)} .
$$

The next corollary is a generalization of [21, Theorem 84, page 178].

Corollary 5.4. If $\alpha, m$, and $n \in \mathbb{N}$, then

$$
\sum_{k=1}^{\infty} \frac{c^{(\alpha)}(n, k m)}{k}=0
$$


Proof. The result follows from Theorem 5.3 by taking the limit $s \rightarrow 1^{+}$and using the fact that $\lim _{s \rightarrow 1^{+}}(1 / \zeta(s))=0$.

Additional identities extending [21, Theorems 85, 86, and 87 in Section 18.1] will be derived next.

Theorem 5.5. If $\alpha, n \in \mathbb{N}$ and $s>0$ is real, then

$$
\frac{\phi_{s}^{(\alpha)}(n)(\zeta(s+1))^{\alpha}}{n^{s}}=\sum_{j=1}^{\infty} \frac{c(n, j) \mu_{\alpha}(j)}{j^{s+1}} \prod_{p \mid j} \frac{1+\sum_{i \geq 1}\left(\mu_{\alpha}\left(p^{v_{p}(j)+i}\right) / \mu_{\alpha}\left(p^{v_{p}(j)}\right) p^{i(s+1)}\right)}{1+\sum_{i \geq 1}\left(\mu_{\alpha}\left(p^{i}\right) / p^{i(s+1)}\right)} .
$$

Proof. Following the proof of Theorem 5.1, taking

$$
F(u, v)=\mu_{\alpha}(u) v^{s}, \quad D(n)=\sum_{u v=n} F(u, v)=\sum_{u v=n} \mu_{\alpha}(u) v^{s}=\phi_{s}^{(\alpha)}(n),
$$

we obtain

$$
D(n)=c(n, 1) \sum_{x=1}^{[t]} \frac{1}{x} F\left(x, \frac{n}{x}\right)+c(n, 2) \sum_{x=1}^{[t / 2]} \frac{1}{2 x} F\left(2 x, \frac{n}{2 x}\right)+\cdots
$$

Thus,

$$
\frac{\phi_{s}^{(\alpha)}(n)}{n^{s}}=c(n, 1) \sum_{x=1}^{[t]} \frac{\mu_{\alpha}(x)}{x^{s+1}}+c(n, 2) \sum_{x=1}^{[t / 2]} \frac{\mu_{\alpha}(2 x)}{(2 x)^{s+1}}+\cdots
$$

where $t$ is real and $\geq n$. Since $s>0$, letting $t \rightarrow \infty$ we get

$$
\frac{\phi_{s}^{(\alpha)}(n)}{n^{s}}=c(n, 1) \sum_{x=1}^{\infty} \frac{\mu_{\alpha}(x)}{x^{s+1}}+c(n, 2) \sum_{x=1}^{\infty} \frac{\mu_{\alpha}(2 x)}{(2 x)^{s+1}}+\cdots .
$$

Applying the result of Theorem 2.9 to the right-hand expression, the result follows.

Theorem 5.6. If $r \in \mathbb{N}, \alpha \in \mathbb{C}$, and $s>1$ is real, then

$$
\sum_{n=1}^{\infty} \frac{c^{(\alpha)}(n, r)}{n^{s}}=\zeta(s) \phi_{1-s}^{(\alpha)}(r)
$$

Proof. Since $\sum_{n=1}^{\infty}\left(c^{(\alpha)}(n, r) / n^{s}\right)=\sum_{n=1}^{\infty}\left(1 / n^{s}\right) \sum_{d \mid(n, r)} d \mu_{\alpha}(r / d)$, we have

$$
\begin{aligned}
\sum_{n=1}^{\infty} \frac{c^{(\alpha)}(n, r)}{n^{s}} & =\sum_{d \mid r} d \mu_{\alpha}\left(\frac{r}{d}\right) \sum_{m=1}^{\infty} \frac{1}{(m d)^{s}}=\sum_{m=1}^{\infty} \frac{1}{m^{s}} \sum_{d \mid r} d^{1-s} \mu_{\alpha}\left(\frac{r}{d}\right) \\
& =\zeta(s)\left(\mu_{\alpha} * \zeta_{1-s}\right)(r)=\zeta(s) \phi_{1-s}^{(\alpha)}(r) .
\end{aligned}
$$

Theorems 5.2 and 5.6 are special cases of more general results in [1] and for even more general results, see [7]. 
Theorem 5.7. If $n, r$, and $\alpha \in \mathbb{N}$, then

$$
\sum_{n=1}^{\infty} \frac{c^{(\alpha)}(n, r)}{n}=-\sum_{d \mid n} \mu_{\alpha}\left(\frac{r}{d}\right) \log d
$$

whenever $r$ is $\alpha$-powerful.

Proof. From Theorem 5.6, for real $s>1$, we have

$$
\begin{aligned}
\sum_{n=1}^{\infty} \frac{c^{(\alpha)}(n, r)}{n^{s}} & =\zeta(s) \sum_{d \mid r} d^{1-s} \mu_{\alpha}\left(\frac{r}{d}\right)=\zeta(s) \sum_{d \mid r} \mu_{\alpha}\left(\frac{r}{d}\right) e^{(1-s) \log d} \\
& =\zeta(s) \sum_{d \mid r} \mu_{\alpha}\left(\frac{r}{d}\right)\left\{1+\frac{(1-s) \log d}{1 !}+\frac{(1-s)^{2} \log ^{2} d}{2 !}+\cdots\right\}
\end{aligned}
$$

Since $\lim _{s \rightarrow 1^{+}} \zeta(s)(s-1)=1$ and

$$
\sum_{d \mid r} \mu_{\alpha}\left(\frac{r}{d}\right)=\mu_{\alpha-1}(r)=\prod_{p \mid r}(-1)^{v_{p}(r)}\left(\begin{array}{c}
\alpha-1 \\
v_{p}(r)
\end{array}\right)=0
$$

for $r$ being $\alpha$-powerful, we have

$$
\lim _{s \rightarrow 1} \zeta(s) \phi_{1-s}^{(\alpha)}(r)=\lim _{s \rightarrow 1^{+}} \zeta(s)(1-s) \sum_{d \mid r} \mu_{\alpha}\left(\frac{r}{d}\right) \log d=-\sum_{d \mid r} \mu_{\alpha}\left(\frac{r}{d}\right) \log d,
$$

and the desired result follows.

\section{Inverse and reciprocity law}

Recall that an arithmetic function of one variable $f$ possesses a Dirichlet inverse, $f^{-1}$, if and only if $f(1) \neq 0$. In order to find the inverse of a GRS, which is a function of two variables, we resort to the technique of Haukkanen [9] which involves the use of principal functions. We now briefly review Haukkanen's technique. If $f$ and $g$ are arithmetic functions of one variable, by $f: g$ we mean the arithmetic function of two variables

$$
(f: g)(m, n)=f(m) g(n) \quad(m, n \in \mathbb{N}) .
$$

The Dirichlet convolution of two arithmetic functions $F, G$ of two variables is defined by

$$
(F * * G)(m, n)=\sum_{d \mid m} \sum_{e \mid n} F(d, e) G\left(\frac{m}{d}, \frac{n}{e}\right) .
$$

The identity under the Dirichlet convolution of arithmetic functions of two variables is $I: I$, where $I$ denotes the identity relative to the Dirichlet convolution of arithmetic functions of one variable. The Dirichlet inverse of an arithmetic function, $F$, of two variables is the unique arithmetic function of two variables, $F^{* *-1}$, enjoying the properties that $F^{* *-1} * * F=F * * F^{* *-1}=I: I$. It is easily shown that such Dirichlet inverse exists if 
20 Ramanujan sums via generalized Möbius functions

and only if $F(1,1) \neq 0$, and in such case, we have

$$
(F * * G)^{* *-1}=F^{* *-1} * * G^{* *-1} .
$$

In particular,

$$
(f: g)^{* *-1}=f^{-1}: g^{-1} \text {. }
$$

For an arithmetic function of one variable, $f$, its principal function, $P(f)$, which is an arithmetic function of two variables, is defined as

$$
P(f)(m, n)= \begin{cases}f(n), & \text { if } m=n, \\ 0, & \text { otherwise. }\end{cases}
$$

Clearly, $P(f * g)=P(f) * * P(g)$ and $(P(f))^{* *-1}=P\left(f^{-1}\right)$. The sum of the form

$$
S(m, n)=\sum_{d \mid \operatorname{gcd}(m, n)} f(d) g\left(\frac{m}{d}\right) h\left(\frac{n}{d}\right)
$$

can also be written as

$$
S=P(f) * *(g: h)
$$

If $f(1), g(1)$, and $h(1)$ are all nonzero, then $S$ is invertible and

$$
S^{* *-1}=P\left(f^{-1}\right) * *\left(g^{-1}: h^{-1}\right),
$$

that is,

$$
S^{* *-1}(m, n)=\sum_{d \mid \operatorname{gcd}(m, n)} f^{-1}(d) g^{-1}\left(\frac{m}{d}\right) h^{-1}\left(\frac{n}{d}\right) .
$$

TheOREM 6.1. The Dirichlet inverse of the GRS $c^{(\alpha)}(m, n)$ is

$$
c^{(\alpha) * *-1}=P\left(\zeta_{1}^{-1}\right) * *\left(\mu: \mu_{-\alpha}\right) .
$$

In other words,

$$
c^{(\alpha) * *-1}(m, n)=\sum_{d \mid \operatorname{gcd}(m, n)} \zeta_{1}^{-1}(d) \mu\left(\frac{m}{d}\right) \mu_{-\alpha}\left(\frac{n}{d}\right) .
$$

Proof. Since $c^{(\alpha)}(m, n)=\sum_{d \mid \operatorname{gcd}(m, n)} d \mu_{\alpha}(n / d)$, we have $c^{(\alpha)}=P\left(\zeta_{1}\right) * *\left(\zeta_{0}: \mu_{\alpha}\right)$. Thus,

$$
c^{(\alpha) * *-1}=P\left(\zeta_{1}^{-1}\right) * *\left(\zeta_{0}^{-1}: \mu_{\alpha}^{-1}\right)=P\left(\zeta_{1}^{-1}\right) * *\left(\mu: \mu_{-\alpha}\right) .
$$


Moreover,

$$
\begin{aligned}
c^{(\alpha) * *-1}(m, n) & =\sum_{d \mid m} \sum_{e \mid n} P\left(\zeta_{1}^{-1}\right)(d, e)\left(\mu: \mu_{-\alpha}\right)\left(\frac{m}{d}, \frac{n}{e}\right) \\
& =\sum_{d \mid \operatorname{gcd}(m, n)} \zeta_{1}^{-1}(d) \mu\left(\frac{m}{d}\right) \mu_{-\alpha}\left(\frac{n}{d}\right) .
\end{aligned}
$$

Following [21], a reciprocity law is a relation involving an arithmetic function of two variables which possesses a symmetry in the variables. In the classical case, Ramanujan sums satisfy the following reciprocity law [21, Theorem 88, page 184]: for all $n, r \in \mathbb{N}$, we have

$$
\frac{\mu(\gamma(r))}{r^{*}} c\left(n r^{*}, r\right)=\frac{\mu(\gamma(n))}{n^{*}} c\left(n^{*} r, n\right)
$$

where $\gamma(r)$ is the largest square-free divisor of the integer $r>1, \gamma(1):=1$, and $r^{*}:=$ $r / \gamma(r)$. The validity of this law follows directly from the symmetric identity

$$
\mu(r) c(n, r)=\mu(n) c(r, n)
$$

which holds for square-free integers $n, r$. The following examples show that both (6.14) and (6.15) do not hold for general GRSs.

Examples 6.2. (1) $\left(\mu_{2}(\gamma(8)) / 4\right) c^{(2)}(12 \cdot 4,8)=-1 \neq-4=\left(\mu_{2}(\gamma(12)) / 2\right) c^{(2)}(2 \cdot 8,12)$.

(2) $\mu_{3}(2) c^{(3)}(6,2)=3 \neq 27=\mu_{3}(6) c^{(3)}(2,6)$.

We now investigate when such a reciprocity law exists. Analyzing (6.15), we get the following.

Lemma 6.3. Let $n, r \in \mathbb{N}$ and $\alpha \in \mathbb{C}$. For a square-free $r \in \mathbb{N}$, then

$$
\mu_{\alpha}(r) c^{(\alpha)}(n, r)=\prod_{\substack{p|r \\ p| n}}\left\{\left(\mu_{\alpha}(p)\right)^{2}+p \mu_{\alpha}(p)\right\} \prod_{\substack{p \mid r \\ p \nmid n}}\left(\mu_{\alpha}(p)\right)^{2}
$$

Proof. For a fixed $n \in \mathbb{N}$, since $c^{(\alpha)}(n, r)$ and $\mu_{\alpha}(r)$ are multiplicative functions of $r$, for square-free $r$, we have

$$
\mu_{\alpha}(r) c^{(\alpha)}(n, r)=\prod_{p|r d| \operatorname{gcd}(n, p)} \sum_{\alpha}(p) \mu_{\alpha}\left(\frac{p}{d}\right) d=\prod_{\substack{p|r \\ p| n}}\left\{\left(\mu_{\alpha}(p)\right)^{2}+p \mu_{\alpha}(p)\right\} \prod_{\substack{p \mid r \\ p \nmid n}}\left(\mu_{\alpha}(p)\right)^{2}
$$

From Lemma 6.3, we see clearly that in order to get a relation like (6.15), the nonsymmetric term $\prod_{p \mid r}\left(\mu_{\alpha}(p)\right)^{2}$ must take the same value when $n$ and $r$ are interchanged. This can happen only in two situations, namely,

(1) $\alpha=1$ or 
(2) $n$ and $r$ have the same prime divisors, and the number of primes dividing $n$ but not dividing $r$ is the same as the number of primes dividing $r$ but not dividing $n$. Since the case $\alpha=1$ corresponds to the classical Ramanujan sum and its reciprocity is already well-known [21, Section IX.2], in the rest of this section, we concentrate on deriving a reciprocity law in the case $\alpha \neq 1$. In this situation, to ensure symmetry, the same result as in Lemma 6.3 must also hold for square-free $n$. Taking into account the fact that $\mu_{\alpha}(n)=\mu_{\alpha}(r)$ for $n, r$ both square-free and have the same number of prime factors, we immediately deduce the following.

Proposition 6.4. Let $n, r \in \mathbb{N}$ and $\alpha \in \mathbb{C} \backslash\{1\}$. If $n$ and $r$ are square-free and have the same number of prime factors, then

$$
c^{(\alpha)}(n, r)=c^{(\alpha)}(r, n),
$$

provided $\mu_{\alpha}(r)$ and $\mu_{\alpha}(n)$ are both nonzero.

Our reciprocity law reads now.

Theorem 6.5. Let $\alpha \in \mathbb{C} \backslash\{1\}$. If the positive integers $r$ and $s$ have their unique prime representations of the form

$$
n=p_{1}^{a_{1}} \cdots p_{t}^{a_{t}} q_{11} \cdots q_{1 s}, \quad r=p_{1}^{b_{1}} \cdots p_{t}^{b_{t}} q_{21} \cdots q_{2 s}
$$

where $p_{i}, q_{1 j}, q_{2 k}$ are distinct primes, $a_{i}, b_{j}$ positive integers, $t$, s nonnegative integers, then

$$
\phi_{1}^{(\alpha)}\left(p_{1}^{a_{1}} \cdots p_{t}^{a_{t}}\right) c^{(\alpha)}\left(n r^{*}, r\right)=\phi_{1}^{(\alpha)}\left(p_{1}^{b_{1}} \cdots p_{t}^{b_{t}}\right) c^{(\alpha)}\left(n^{*} r, n\right),
$$

provided that $\phi_{1}^{(\alpha)}\left(q_{11} \cdots q_{1 s}\right)$ and $\phi_{1}^{(\alpha)}\left(q_{21} \cdots q_{2 s}\right)$ are both nonzero.

Proof. Using the above notation, from the shape of their prime factorizations, we easily checked that $\left\langle n r^{*}, r\right\rangle$ is a unitary pair and so Corollary 2.6 yields

$$
c^{(\alpha)}\left(n r^{*}, r\right)=\mu_{\alpha}\left(q_{21} \cdots q_{2 s}\right) \phi_{1}^{(\alpha)}\left(p_{1}^{a_{1}} \cdots p_{t}^{a_{t}}\right),
$$

provided that $\phi_{1}^{(\alpha)}\left(q_{21} \cdots q_{2 s}\right) \neq 0$. Similarly, provided that $\phi_{1}^{(\alpha)}\left(q_{11} \cdots q_{1 s}\right) \neq 0$, we have

$$
c^{(\alpha)}\left(n^{*} r, n\right)=\mu_{\alpha}\left(q_{11} \cdots q_{1 s}\right) \phi_{1}^{(\alpha)}\left(p_{1}^{b_{1}} \cdots p_{t}^{b_{t}}\right) .
$$

Since $\mu_{\alpha}\left(q_{11} \cdots q_{1 s}\right)=\mu_{\alpha}\left(q_{21} \cdots q_{2 s}\right)$, the result follows.

Theorem 6.5 reduces to Proposition 6.4 when both $n$ and $r$ are square-free.

\section{Dependence of GRSs}

It is shown in [16] that the Souriau-Hsu-Möbius functions, $\mu_{\alpha}$, of finitely many parameters $\alpha$, are $\mathbb{C}$-algebraically independent if and only if such parameters are $\mathbb{Z}$-linearly independent. In this section, we ask the same question for GRSs. Since a GRS is a function of two variables, which is multiplicative in the second variable; we look at this question for a GRS considered with respect to this second variable while keeping the first variable fixed. The answer is affirmative in one direction. 
Theorem 7.1. Let $k \in \mathbb{N}, n \in \mathbb{N}_{0}$ be fixed integers, and $\alpha_{1}, \ldots, \alpha_{k} \in \mathbb{C}$. If $\alpha_{1}, \ldots, \alpha_{k}$ are $\mathbb{Z}$ linearly independent, then $c^{\left(\alpha_{1}\right)}(n, \cdot), \ldots, c^{\left(\alpha_{k}\right)}(n, \cdot)$ are $\mathbb{C}$-algebraically independent.

Proof. Assume to the contrary that $c^{\left(\alpha_{1}\right)}(n, \cdot), \ldots, c^{\left(\alpha_{k}\right)}(n, \cdot)$ are $\mathbb{C}$-algebraically dependent. By [17, Theorem 4$]$, there exist $j_{1}, \ldots, j_{k} \in \mathbb{Z}$ not all zero such that

$$
Q=\left(c^{\left(\alpha_{1}\right)}(n, \cdot)\right)^{j_{1}} * \cdots *\left(c^{\left(\alpha_{k}\right)}(n, \cdot)\right)^{j_{k}}
$$

vanishes on $\mathbb{N}^{\prime}=\mathbb{N} \backslash$ a semigroup generated by finitely many primes. Let $p \in \mathbb{N}^{\prime}$ be prime such that $p \nmid n$. Then

$$
\begin{aligned}
0=Q(p) & =\left(c^{\left(\alpha_{1}\right)}(n, \cdot)\right)^{j_{1}} * \cdots *\left(c^{\left(\alpha_{k}\right)}(n, \cdot)\right)^{j_{k}}(p) \\
& =j_{1} c^{\left(\alpha_{1}\right)}(n, p)+\cdots+j_{k} c^{\left(\alpha_{k}\right)}(n, p) \\
& =-j_{1} \alpha_{1}-\cdots-j_{k} \alpha_{k} .
\end{aligned}
$$

Since $\alpha_{1}, \ldots, \alpha_{k}$ are $\mathbb{Z}$-linearly independent, we must have $j_{1}=\cdots=j_{k}=0$, which is a contradiction.

Theorem 7.1 does not hold if the $\mathbb{C}$-algebraically independence is replaced by independence over a subring of the ring of all arithmetic functions because by the result (1) of Lemma 1.2, we know that $c^{(\alpha-1)}(m, n)=\left(c^{(\alpha)}(m, \cdot) * \zeta_{0}\right)(n)$, while $\alpha$ and $\alpha-1$ are $\mathbb{Z}$ linearly independent for irrational $\alpha$.

The question about the converse of Theorem 7.1 needs more analysis as the answer depends closely on the first variable. For at least one specific value of the first variable, the converse is true.

Theorem 7.2. Let $k \in \mathbb{N}$ and $\alpha_{1}, \ldots, \alpha_{k} \in \mathbb{C}$. If $c^{\left(\alpha_{1}\right)}(1, \cdot), \ldots, c^{\left(\alpha_{k}\right)}(1, \cdot)$ are $\mathbb{C}$-algebraically independent, then $\alpha_{1}, \ldots, \alpha_{k}$ are $\mathbb{Z}$-linearly independent.

Proof. Suppose that $\alpha_{1}, \ldots, \alpha_{k}$ are $\mathbb{Z}$-linearly dependent. We may assume without loss of generality that there are $i_{1}, \ldots, i_{k-1} \in \mathbb{Z}$ such that $\alpha_{k}=i_{1} \alpha_{1}+\cdots+i_{k-1} \alpha_{k-1}$. By the result (5) of Lemma 1.2,

$$
\begin{aligned}
c^{\left(\alpha_{k}\right)}(1, \cdot) & =\mu_{\alpha_{k}}=\mu_{i_{1} \alpha_{1}+\cdots+i_{k-1} \alpha_{k-1}}=\mu_{i_{1} \alpha_{1}} * \cdots * \mu_{i_{k-1} \alpha_{k-1}} \\
& =\mu_{\alpha_{1}}^{i_{1}} * \cdots * \mu_{\alpha_{k-1}}^{i_{k-1}}=\left(c^{\left(\alpha_{1}\right)}(1, \cdot)\right)^{i_{1}} * \cdots *\left(c^{\left(\alpha_{k-1}\right)}(1, \cdot)\right)^{i_{k-1}},
\end{aligned}
$$

and so $c^{\left(\alpha_{1}\right)}(1, \cdot), \ldots, c^{\left(\alpha_{k}\right)}(1, \cdot)$ are $\mathbb{C}$-algebraically dependent.

In general, the converse of Theorem 7.1 is not true as shown in the following example. Take $n=2, \alpha=1$, and $\beta=2$. Clearly, $\alpha$ and $\beta$ are $\mathbb{Z}$-linearly dependent. Let $q$ be an odd prime. Using an algebraic independence test of Shapiro and Sparer [20, Theorem 5], by considering the Jacobian

$$
J\left(c^{(1)}(2, \cdot), c^{(2)}(2, \cdot) ; 2, q\right)(1)=\left|\begin{array}{ll}
c^{(1)}(2,2) v_{2}(2) & c^{(2)}(2,2) v_{2}(2) \\
c^{(1)}(2, q) v_{q}(q) & c^{(2)}(2, q) v_{q}(q)
\end{array}\right|=\left|\begin{array}{cc}
1 & 0 \\
-1 & -2
\end{array}\right|=-2 \neq 0,
$$


we conclude that $c^{(1)}(2, \cdot)$ and $c^{(2)}(2, \cdot)$ are $\mathbb{C}$-algebraically independent. This example also shows that the converse of Popken [17, Theorem 4], the result that was used in the proof of Theorem 7.1 above which states that if multiplicative functions $f_{1}, \ldots, f_{n}$ are $\mathbb{C}$ algebraically dependent, then there exist $i_{1}, \ldots, i_{n} \in \mathbb{Z}$, not all zero such that $f_{1}^{i_{1}} * \cdots *$ $f_{n}^{i_{n}}=0$ on the set $\mathbb{N}^{\prime}=\mathbb{N} \backslash$ semigroup generated by finitely many primes, is not generally true.

Since the converse of Theorem 7.1 depends on the first variable, instead of asking for the converse relative to GRSs with a fixed first variable, it seems more appropriate to consider the mean value with respect to the first variable of any GRS and in this case we have indeed a valid converse.

Theorem 7.3. Let $k, r \in \mathbb{N}$ be fixed. Then $1, \alpha_{1}, \ldots, \alpha_{k} \in \mathbb{C}$ are $\mathbb{Z}$-linearly independent if and only if $M(c(\cdot, r)), M\left(c^{\left(\alpha_{1}\right)}(\cdot, r)\right), \ldots, M\left(c^{\left(\alpha_{k}\right)}(\cdot, r)\right)$ are $\mathbb{C}$-algebraically independent.

Proof. This is an immediate consequence of Theorem 4.1 above and [16, Theorem 11].

\section{Applications}

In this section, we give two applications of GRSs. First, we extend, in two different ways [12, Theorem 1] which gives an interesting characterization of completely multiplicative functions using Ramanujan sums. Second, we discuss a relation between a GRS and the solutions of certain congruences established by Wang and Hsu [24].

THeORem 8.1. Let $f$ be a nonzero multiplicative arithmetic function whose associated Dirichlet series is $F(s)=\sum_{n \geq 1} f(n) / n^{s}$. Let $c^{(\alpha)}(n, m)$ be the GRS of order $\alpha \in \mathbb{C} \backslash\{0\}$.

(i) If $f$ is completely multiplicative, then for each $m \in \mathbb{N}$,

$$
\sum_{n \geq 1} \frac{f(n) c^{(\alpha)}(n, m)}{n^{s}}=(F(s))^{\alpha} \sum_{n \geq 1} \frac{f(n) M^{(\alpha)}(m, n)}{n^{s}}
$$

where $M^{(\alpha)}(m, n)=\sum_{r \mid \operatorname{gcd}(m, n)} r \mu_{\alpha}(m / r) \mu_{\alpha-1}(n / r)$.

(ii) If $f$ satisfies (8.1) for all $m \in \mathbb{N}$, then $f$ is completely multiplicative.

Proof. (i) Assume $f$ is completely multiplicative. Using $f^{-\alpha}=\mu_{\alpha} f$, we get

$$
(F(s))^{-\alpha}=\sum_{n \geq 1} \frac{\left(\mu_{\alpha} f\right)(n)}{n^{s}} .
$$

Thus

$$
\begin{aligned}
\left(\mu_{\alpha} f\right) *\left(f c^{(\alpha)}(\cdot, m)\right)(n) & =\sum_{d \mid n} \mu_{\alpha}(d) f(d) f\left(\frac{n}{d}\right) c^{(\alpha)}\left(\frac{n}{d}, m\right)=f(n) \sum_{d \mid n} \mu_{\alpha}(d) \sum_{\substack{r|m \\
r| n / d}} r \mu_{\alpha}\left(\frac{m}{r}\right) \\
& =f(n) \sum_{\substack{r|m \\
r| n}} r \mu_{\alpha}\left(\frac{m}{r}\right) \sum_{d \mid n / r} \mu_{\alpha}(d)=f(n) \sum_{\substack{r|m \\
r| n}} r \mu_{\alpha}\left(\frac{m}{r}\right) \mu_{\alpha-1}\left(\frac{n}{r}\right)
\end{aligned}
$$


that is,

$$
(F(s))^{-\alpha} \sum_{n \geq 1} \frac{f(n) c^{(\alpha)}(n, m)}{n^{s}}=\sum_{n \geq 1} \frac{f(n)}{n^{s}} \sum_{\substack{r|m \\ r| n}} r \mu_{\alpha}\left(\frac{m}{r}\right) \mu_{\alpha-1}\left(\frac{n}{r}\right)
$$

(ii) Assume $f$ satisfies (8.1). Then

$$
\left(f^{-\alpha} * f c^{(\alpha)}(\cdot, m)\right)(n)=f(n) \sum_{r \mid g c d(m, n)} r \mu_{\alpha}\left(\frac{m}{r}\right) \mu_{\alpha-1}\left(\frac{n}{r}\right) .
$$

We assert that $f^{-\alpha}\left(p^{a}\right)=\left(\mu_{\alpha} f\right)\left(p^{a}\right)$ for all primes $p$ and $a \in \mathbb{N}$.

To see this, when $a=1$, substituting $m=1$ and $n=p$ in (8.5), the left-hand side is

$$
f^{-\alpha}(1) f(p) c^{(\alpha)}(p, 1)+f^{-\alpha}(p) f(1) c^{(\alpha)}(1,1)=f(p)+f^{-\alpha}(p)
$$

while the right-hand side is

$$
f(p) \sum_{r \mid(1, p)} r \mu_{\alpha}\left(\frac{1}{r}\right) \mu_{\alpha-1}\left(\frac{p}{r}\right)=-(\alpha-1) f(p) .
$$

Equating both sides, we get $f^{-\alpha}(p)=-\alpha f(p)=\left(\mu_{\alpha} f\right)(p)$.

When $a=2$, substituting $n=p^{2}$ and $m=p^{i}\left(i \in \mathbb{N}_{0}\right)$, in (8.5), the left-hand side is

$$
\begin{aligned}
\left(f^{-\alpha} * f c^{(\alpha)}\left(\cdot, p^{i}\right)\right)\left(p^{2}\right)= & (-1)^{i}\left(\begin{array}{c}
\alpha \\
i
\end{array}\right) f^{-\alpha}\left(p^{2}\right)+(-1)^{i+1}\left(\begin{array}{c}
\alpha \\
1
\end{array}\right) f(p) f(p)\left[\left(\begin{array}{c}
\alpha \\
i
\end{array}\right)-\left(\begin{array}{c}
\alpha \\
i-1
\end{array}\right) p\right] \\
& +(-1)^{i}\left(\begin{array}{l}
\alpha \\
0
\end{array}\right) f(1) f\left(p^{2}\right)\left[\left(\begin{array}{c}
\alpha \\
i
\end{array}\right)-\left(\begin{array}{c}
\alpha \\
i-1
\end{array}\right) p+\left(\begin{array}{c}
\alpha \\
i-2
\end{array}\right) p^{2}\right]
\end{aligned}
$$

while the right-hand side is

$$
\begin{aligned}
f\left(p^{2}\right) & \sum_{r \mid \operatorname{gcd}\left(p^{i}, p^{2}\right)} r \mu_{\alpha}\left(\frac{p^{i}}{r}\right) \mu_{\alpha-1}\left(\frac{p^{2}}{r}\right) \\
= & (-1)^{i} f\left(p^{2}\right)\left[\left(\begin{array}{c}
\alpha \\
i
\end{array}\right)\left(\begin{array}{c}
\alpha-1 \\
2
\end{array}\right)+p\left(\begin{array}{c}
\alpha \\
i-1
\end{array}\right)\left(\begin{array}{c}
\alpha-1 \\
1
\end{array}\right)+p^{2}\left(\begin{array}{c}
\alpha \\
i-2
\end{array}\right)\right]
\end{aligned}
$$

Equating both sides leads to

$$
\begin{aligned}
\left(\begin{array}{c}
\alpha \\
i
\end{array}\right) f^{-\alpha}\left(p^{2}\right)= & f\left(p^{2}\right)\left[\left(\begin{array}{c}
\alpha \\
i
\end{array}\right)\left(\begin{array}{c}
\alpha-1 \\
2
\end{array}\right)+p\left(\begin{array}{c}
\alpha \\
i-1
\end{array}\right)\left(\begin{array}{c}
\alpha-1 \\
1
\end{array}\right)+p^{2}\left(\begin{array}{c}
\alpha \\
i-2
\end{array}\right)\right] \\
& +\left(\begin{array}{c}
\alpha \\
1
\end{array}\right) f(p) f(p)\left[\left(\begin{array}{c}
\alpha \\
i
\end{array}\right)-\left(\begin{array}{c}
\alpha \\
i-1
\end{array}\right) p\right] \\
& +(-1)\left(\begin{array}{c}
\alpha \\
0
\end{array}\right) f(1) f\left(p^{2}\right)\left[\left(\begin{array}{c}
\alpha \\
i
\end{array}\right)-\left(\begin{array}{c}
\alpha \\
i-1
\end{array}\right) p+\left(\begin{array}{c}
\alpha \\
i-2
\end{array}\right) p^{2}\right] .
\end{aligned}
$$


Putting $i=0$ in (8.10) and simplifying, we get

$$
\left(\begin{array}{l}
\alpha \\
1
\end{array}\right) f^{-\alpha}\left(p^{2}\right)=f\left(p^{2}\right)\left[\left(\begin{array}{c}
\alpha \\
1
\end{array}\right)\left(\begin{array}{c}
\alpha-1 \\
2
\end{array}\right)-\left(\begin{array}{l}
\alpha \\
1
\end{array}\right)\right]+\left(\begin{array}{l}
\alpha \\
1
\end{array}\right)\left(\begin{array}{l}
\alpha \\
1
\end{array}\right) f(p) f(p) .
$$

Putting $i=1$ in $(8.10)$, we get

$$
\begin{aligned}
\left(\begin{array}{l}
\alpha \\
1
\end{array}\right) f^{-\alpha}\left(p^{2}\right)= & f\left(p^{2}\right)\left[\left(\begin{array}{l}
\alpha \\
1
\end{array}\right)\left(\begin{array}{c}
\alpha-1 \\
2
\end{array}\right)+p\left(\begin{array}{l}
\alpha \\
0
\end{array}\right)\left(\begin{array}{c}
\alpha-1 \\
1
\end{array}\right)\right] \\
& +\left(\begin{array}{c}
\alpha \\
1
\end{array}\right) f(p) f(p)\left[\left(\begin{array}{l}
\alpha \\
1
\end{array}\right)-\left(\begin{array}{l}
\alpha \\
0
\end{array}\right) p\right]+(-1) f\left(p^{2}\right)\left[\left(\begin{array}{l}
\alpha \\
1
\end{array}\right)-\left(\begin{array}{l}
\alpha \\
0
\end{array}\right) p\right] .
\end{aligned}
$$

Equating (8.11) and (8.12) and simplifying, we get $\left(\begin{array}{l}\alpha \\ 1\end{array}\right) f\left(p^{2}\right)=\left(\begin{array}{l}\alpha \\ 1\end{array}\right) f(p) f(p)$. Since $\alpha \neq 0$, this gives $f\left(p^{2}\right)=f(p) f(p)$. Substituting this into (8.11), we get

$$
f^{-\alpha}\left(p^{2}\right)=f\left(p^{2}\right)\left[\left(\begin{array}{c}
\alpha-1 \\
2
\end{array}\right)+\left(\begin{array}{c}
\alpha-1 \\
1
\end{array}\right)\right]=f\left(p^{2}\right)\left(\begin{array}{l}
\alpha \\
2
\end{array}\right)=\left(\mu_{\alpha} f\right)\left(p^{2}\right) .
$$

For $a \in \mathbb{N}$, assume that

$$
f^{-\alpha}\left(p^{j}\right)=\left(\mu_{\alpha} f\right)\left(p^{j}\right), \quad f\left(p^{j}\right)=f(p)^{j} \quad \text { for } 1 \leq j \leq a-1 .
$$

Then for fixed $m \in \mathbb{N}$, we have

$$
\begin{aligned}
\left(f^{-\alpha} * f c^{(\alpha)}(\cdot, m)\right)\left(p^{a}\right)= & f^{-\alpha}\left(p^{a}\right) \mu_{\alpha}(m)+\left(\begin{array}{c}
\alpha \\
a-1
\end{array}\right)(-1)^{a-1} f\left(p^{a-1}\right) f(p) c^{(\alpha)}(p, m)+\cdots \\
& +\left(\begin{array}{c}
\alpha \\
1
\end{array}\right)(-1) f(p) f\left(p^{a-1}\right) c^{(\alpha)}\left(p^{a-1}, m\right)+f\left(p^{a}\right) c^{(\alpha)}\left(p^{a}, m\right) .
\end{aligned}
$$

Putting $m=p^{i}(1 \leq i \leq a-1)$ in (8.15) and simplifying, we get

$$
\begin{aligned}
f\left(p^{a}\right) & \sum_{r \mid \operatorname{gcd}\left(p^{i}, p^{a}\right)} r \mu_{\alpha}\left(\frac{p^{i}}{r}\right) \mu_{\alpha-1}\left(\frac{p^{a}}{r}\right) \\
& =(-1)^{a+i} f\left(p^{a}\right)\left[\left(\begin{array}{c}
\alpha \\
i
\end{array}\right)\left(\begin{array}{c}
\alpha-1 \\
a
\end{array}\right)+p\left(\begin{array}{c}
\alpha \\
i-1
\end{array}\right)\left(\begin{array}{c}
\alpha-1 \\
a-1
\end{array}\right)+\cdots+p^{i}\left(\begin{array}{c}
\alpha \\
0
\end{array}\right)\left(\begin{array}{c}
\alpha-1 \\
a-i
\end{array}\right)\right] .
\end{aligned}
$$


Putting $n=p^{a}$ in (8.5) and simplifying, we get

$$
\begin{aligned}
\left(\begin{array}{c}
\alpha \\
i
\end{array}\right) f^{-\alpha}\left(p^{a}\right)= & (-1)^{a} f\left(p^{a}\right)\left[\left(\begin{array}{c}
\alpha \\
i
\end{array}\right)\left(\begin{array}{c}
\alpha-1 \\
a
\end{array}\right)+p\left(\begin{array}{c}
\alpha \\
i-1
\end{array}\right)\left(\begin{array}{c}
\alpha-1 \\
a-1
\end{array}\right)+\cdots+p^{i}\left(\begin{array}{c}
\alpha \\
0
\end{array}\right)\left(\begin{array}{c}
\alpha-1 \\
a-i
\end{array}\right)\right] \\
& +(-1)^{a}\left(\begin{array}{c}
\alpha \\
a-1
\end{array}\right) f\left(p^{a-1}\right) f(p)\left[\left(\begin{array}{c}
\alpha \\
i
\end{array}\right)-\left(\begin{array}{c}
\alpha \\
i-1
\end{array}\right) p\right]+\cdots \\
& +(-1)^{2}\left(\begin{array}{c}
\alpha \\
1
\end{array}\right) f(p) f\left(p^{a-1}\right)\left[\left(\begin{array}{c}
\alpha \\
i
\end{array}\right)-\left(\begin{array}{c}
\alpha \\
i-1
\end{array}\right) p+\cdots+(-1)^{a-1}\left(\begin{array}{c}
\alpha \\
i-a+1
\end{array}\right) p^{a-1}\right] \\
& +(-1)\left(\begin{array}{c}
\alpha \\
0
\end{array}\right) f\left(p^{a}\right)\left[\left(\begin{array}{c}
\alpha \\
i
\end{array}\right)-\left(\begin{array}{c}
\alpha \\
i-1
\end{array}\right) p+\cdots+(-1)^{a}\left(\begin{array}{c}
\alpha \\
i-a
\end{array}\right) p^{a}\right] .
\end{aligned}
$$

Putting $i=0$ in (8.17), we get

$$
\begin{aligned}
\left(\begin{array}{l}
\alpha \\
0
\end{array}\right) f^{-\alpha}\left(p^{a}\right)= & (-1)^{a} f\left(p^{a}\right)\left(\begin{array}{c}
\alpha \\
0
\end{array}\right)\left(\begin{array}{c}
\alpha-1 \\
a
\end{array}\right)+(-1)^{a} f\left(p^{a-1}\right) f(p)\left(\begin{array}{c}
\alpha \\
a-1
\end{array}\right)\left(\begin{array}{l}
\alpha \\
0
\end{array}\right)+\cdots \\
& +(-1)^{2} f(p) f\left(p^{a-1}\right)\left(\begin{array}{l}
\alpha \\
1
\end{array}\right)\left(\begin{array}{l}
\alpha \\
0
\end{array}\right)+(-1) f\left(p^{a}\right)\left(\begin{array}{l}
\alpha \\
0
\end{array}\right)\left(\begin{array}{l}
\alpha \\
0
\end{array}\right) .
\end{aligned}
$$

Multiplying (8.18) by $\alpha$, we get

$$
\begin{aligned}
\left(\begin{array}{c}
\alpha \\
1
\end{array}\right) f^{-\alpha}\left(p^{a}\right)= & (-1)^{a} f\left(p^{a}\right)\left(\begin{array}{c}
\alpha \\
1
\end{array}\right)\left(\begin{array}{c}
\alpha-1 \\
a
\end{array}\right)+(-1)^{a} f\left(p^{a-1}\right) f(p)\left(\begin{array}{c}
\alpha \\
a-1
\end{array}\right)\left(\begin{array}{l}
\alpha \\
1
\end{array}\right) \\
& +(-1)^{2} f(p) f\left(p^{a-1}\right)\left(\begin{array}{c}
\alpha \\
1
\end{array}\right)\left(\begin{array}{l}
\alpha \\
1
\end{array}\right)+(-1) f\left(p^{a}\right)\left(\begin{array}{l}
\alpha \\
0
\end{array}\right)\left(\begin{array}{l}
\alpha \\
1
\end{array}\right) .
\end{aligned}
$$

Taking $i=1$ in (8.17), we get

$$
\begin{aligned}
\left(\begin{array}{c}
\alpha \\
1
\end{array}\right) f^{-\alpha}\left(p^{a}\right)= & (-1)^{a} f\left(p^{a}\right)\left[\left(\begin{array}{l}
\alpha \\
1
\end{array}\right)\left(\begin{array}{c}
\alpha-1 \\
a
\end{array}\right)+p\left(\begin{array}{l}
\alpha \\
0
\end{array}\right)\left(\begin{array}{c}
\alpha-1 \\
a-1
\end{array}\right)\right] \\
& +(-1)^{a}\left(\begin{array}{c}
\alpha \\
a-1
\end{array}\right) f\left(p^{a-1}\right) f(p)\left[\left(\begin{array}{l}
\alpha \\
1
\end{array}\right)-\left(\begin{array}{c}
\alpha \\
0
\end{array}\right) p\right]+\cdots \\
& +(-1)\left(\begin{array}{c}
\alpha \\
0
\end{array}\right) f\left(p^{a}\right)\left[\left(\begin{array}{c}
\alpha \\
1
\end{array}\right)-\left(\begin{array}{l}
\alpha \\
0
\end{array}\right) p\right] .
\end{aligned}
$$

Subtracting (8.19) from (8.20) and simplifying, we arrive at

$$
f\left(p^{a}\right)\left[\left(\begin{array}{l}
\alpha-1 \\
a-1
\end{array}\right)+(-1)^{a}\left(\begin{array}{l}
\alpha \\
0
\end{array}\right)\right]=f(p)^{a}\left[\left(\begin{array}{c}
\alpha \\
a-1
\end{array}\right)-\left(\begin{array}{c}
\alpha \\
a-2
\end{array}\right)+\cdots+(-1)^{a}\left(\begin{array}{l}
\alpha \\
1
\end{array}\right)\right] .
$$


Now we need yet another identity

$$
\left(\begin{array}{c}
\alpha-1 \\
a-1
\end{array}\right)=\left(\begin{array}{c}
\alpha \\
a-1
\end{array}\right)-\left(\begin{array}{c}
\alpha \\
a-2
\end{array}\right)+\cdots+(-1)^{a}\left(\begin{array}{l}
\alpha \\
1
\end{array}\right)+(-1)^{a+1}\left(\begin{array}{l}
\alpha \\
0
\end{array}\right)
$$

which follows from iterating the identity $\left(\begin{array}{c}\alpha-1 \\ a-1\end{array}\right)=\left(\begin{array}{c}\alpha \\ a-1\end{array}\right)-\left(\begin{array}{c}\alpha-1 \\ a-2\end{array}\right)$. Using this identity with (8.21), we get

$$
f\left(p^{a}\right)\left[\left(\begin{array}{l}
\alpha-1 \\
a-1
\end{array}\right)+(-1)^{a}\left(\begin{array}{l}
\alpha \\
0
\end{array}\right)\right]=f(p)^{a}\left[\left(\begin{array}{l}
\alpha-1 \\
a-1
\end{array}\right)+(-1)^{a}\right] .
$$

If $\left(\begin{array}{c}\alpha-1 \\ a-1\end{array}\right) \neq(-1)^{a-1}$, then $f\left(p^{a}\right)=f(p)^{a}$. Substituting this last relation into (8.18), we get

$$
f^{-\alpha}\left(p^{a}\right)=(-1)^{a} f(p)^{a}\left[\left(\begin{array}{c}
\alpha-1 \\
a
\end{array}\right)+\left(\begin{array}{c}
\alpha-1 \\
a-1
\end{array}\right)\right]=(-1)^{a} f(p)^{a}\left(\begin{array}{l}
\alpha \\
a
\end{array}\right)=\left(\mu_{\alpha} f\right)\left(p^{a}\right) .
$$

Since $f$ and $\mu_{\alpha}$ are multiplicative, then $f^{-\alpha}$ and $\mu_{\alpha} f$ are also multiplicative. We have shown then by induction that $f^{-\alpha}\left(p^{a}\right)=\left(\mu_{\alpha} f\right)\left(p^{a}\right)$ and $f\left(p^{a}\right)=f(p)^{a}$ for any prime $p$ and $a \in \mathbb{N}$, that is, that $f^{-\alpha}=\mu_{\alpha} f$ and $f$ is completely multiplicative.

If $\left(\begin{array}{c}\alpha-1 \\ a-1\end{array}\right)=(-1)^{a-1}$, then both $\alpha-a+1$ and $a-1$ are nonzero. Thus $\left(\begin{array}{c}\alpha-1 \\ a-2\end{array}\right) \neq(-1)^{a}$. Substituting $i=2$ in (8.17) and simplifying, we get

$$
\begin{aligned}
\left(\begin{array}{l}
\alpha \\
2
\end{array}\right) f^{-\alpha}\left(p^{a}\right) \\
=\left(\begin{array}{l}
\alpha \\
2
\end{array}\right)\left[(-1)^{a} f\left(p^{a}\right)\left(\begin{array}{c}
\alpha-1 \\
a
\end{array}\right)+(-1)^{a} f\left(p^{a-1}\right) f(p)\left(\begin{array}{c}
\alpha \\
a-1
\end{array}\right)+\cdots+(-1) f\left(p^{a}\right)\left(\begin{array}{l}
\alpha \\
0
\end{array}\right)\right] \\
+(-1)^{a} f\left(p^{a}\right) p\left[\left(\begin{array}{l}
\alpha \\
1
\end{array}\right)\left(\begin{array}{c}
\alpha-1 \\
a-1
\end{array}\right)+p\left(\begin{array}{l}
\alpha \\
0
\end{array}\right)\left(\begin{array}{c}
\alpha-1 \\
a-2
\end{array}\right)+\left(\begin{array}{c}
\alpha \\
1
\end{array}\right)\left(\begin{array}{l}
\alpha \\
0
\end{array}\right)(-1)^{a}-(-1)^{a} p\left(\begin{array}{l}
\alpha \\
0
\end{array}\right)\right] \\
\\
+f(p)^{a}\left\{-\left(\begin{array}{c}
\alpha \\
1
\end{array}\right) p(-1)^{a}\left[\left(\begin{array}{c}
\alpha \\
a-1
\end{array}\right)-\left(\begin{array}{c}
\alpha \\
a-2
\end{array}\right)+\left(\begin{array}{c}
\alpha \\
a-3
\end{array}\right)-\cdots+(-1)^{a}\left(\begin{array}{l}
\alpha \\
1
\end{array}\right)\right]\right. \\
\left.+\left(\begin{array}{c}
\alpha \\
0
\end{array}\right) p^{2}(-1)^{a-1}\left[\left(\begin{array}{c}
\alpha \\
a-2
\end{array}\right)-\left(\begin{array}{c}
\alpha \\
a-3
\end{array}\right)+\cdots+(-1)^{a+1}\left(\begin{array}{l}
\alpha \\
1
\end{array}\right)\right]\right\} .
\end{aligned}
$$

Multiplying (8.18) by $\left(\begin{array}{l}\alpha \\ 2\end{array}\right)$, we get

$$
\begin{gathered}
\left(\begin{array}{l}
\alpha \\
2
\end{array}\right) f^{-\alpha}\left(p^{a}\right)=\left(\begin{array}{l}
\alpha \\
2
\end{array}\right)\left[(-1)^{a} f\left(p^{a}\right)\left(\begin{array}{c}
\alpha-1 \\
a
\end{array}\right)+(-1)^{a} f\left(p^{a-1}\right) f(p)\left(\begin{array}{c}
\alpha \\
a-1
\end{array}\right)\right. \\
\left.+\cdots+(-1) f\left(p^{a}\right)\left(\begin{array}{l}
\alpha \\
0
\end{array}\right)\right] .
\end{gathered}
$$


Equating (8.25) and (8.26) yields

$$
\begin{aligned}
& (-1)^{a} f\left(p^{a}\right)\left\{\left(\begin{array}{l}
\alpha \\
1
\end{array}\right)\left[\left(\begin{array}{l}
\alpha-1 \\
a-1
\end{array}\right)+(-1)^{a}\right]+p\left[\left(\begin{array}{l}
\alpha-1 \\
a-2
\end{array}\right)-(-1)^{a}\right]\right\} \\
& =(-1)^{a} f(p)^{a}\left\{\left(\begin{array}{l}
\alpha \\
1
\end{array}\right)\left[\left(\begin{array}{l}
\alpha-1 \\
a-1
\end{array}\right)+(-1)^{a}\right]+p\left[\left(\begin{array}{l}
\alpha-1 \\
a-2
\end{array}\right)-(-1)^{a}\right]\right\} .
\end{aligned}
$$

Since $\left(\begin{array}{l}\alpha-1 \\ a-1\end{array}\right)=(-1)^{a-1}$, and $\left(\begin{array}{c}\alpha-1 \\ a-2\end{array}\right) \neq(-1)^{a}$, we get $f\left(p^{a}\right)=f(p)^{a}$, that is, $f$ is completely multiplicative.

There is another proof of (ii) when $\alpha(\neq 0)$ is not an odd integer $\geq 3$ using [14, Theorem 12] which runs as follows: since $f$ satisfies (8.1), we have as before

$$
\left(f^{-\alpha} * f c^{(\alpha)}(\cdot, m)\right)(n)=f(n) \sum_{r \mid \operatorname{gcd}(m, n)} r \mu_{\alpha}\left(\frac{m}{r}\right) \mu_{\alpha-1}\left(\frac{n}{r}\right) .
$$

Choose $m \in \mathbb{N}$ such that $\operatorname{gcd}(m, n)=1$ and $\mu_{\alpha}(m) \neq 0$. From

$$
\begin{aligned}
&\left(f^{-\alpha} * f c^{\alpha}(\cdot, m)\right)(n)=\sum_{d \mid n} f^{-\alpha}(d) f\left(\frac{n}{d}\right) c^{(\alpha)}\left(\frac{n}{d}, m\right) \\
&=\sum_{d \mid n} f^{-\alpha}(d) f\left(\frac{n}{d}\right) \mu_{\alpha}(m)=\mu_{\alpha}(m) f^{-(\alpha-1)}(n), \\
& f(n) \sum_{r \mid \operatorname{gcd}(m, n)=1} r \mu_{\alpha}\left(\frac{m}{r}\right) \mu_{\alpha-1}\left(\frac{n}{r}\right)=f(n) \mu_{\alpha}(m) \mu_{\alpha-1}(n)=\mu_{\alpha}(m)\left(\mu_{\alpha-1} f\right)(n),
\end{aligned}
$$

we deduce

$$
\mu_{\alpha}(m) f^{-(\alpha-1)}(n)=\mu_{\alpha}(m)\left(\mu_{\alpha-1} f\right)(n)
$$

Thus $\mu_{\alpha}(m) \neq 0$ implies $f^{-(\alpha-1)}=\mu_{\alpha-1} f$, and so $f$ is completely multiplicative by [14, Theorem 1.2].

Theorem 8.1 (ii) is false when $\alpha=0$, for then (8.1) gives

$$
\left(f c^{(0)}(\cdot, m)\right)(n)=f(n) \sum_{r \mid \operatorname{gcd}(m, n)} r \mu_{0}\left(\frac{m}{r}\right) \mu_{-1}\left(\frac{n}{r}\right),
$$

that is, $f(n) \sum_{d \mid \operatorname{gcd}(n, m)} d I(m / d)=f(n) \sum_{r \mid \operatorname{gcd}(m, n)} r I(m / r)$, which holds for any arithmetic function $f$.

In Theorem 8.1, putting $f=\zeta_{0}$ so that $F=\zeta(s)$, we obtain

$$
(\zeta(s))^{-\alpha} \sum_{n \geq 1} \frac{c^{(\alpha)}(n, m)}{n^{s}}=\sum_{n=1}^{\infty} \frac{M^{(\alpha)}(m, n)}{n^{s}},
$$

which seems remarkably similar to Theorems 5.2 and 5.3. 
The function $M^{(\alpha)}(m, n)$ in Theorem 8.1 is interesting because it is an example of an arithmetic function in two variables which is multiplicative. Recall that an arithmetic function $f(m, n)$ in two variables is said to be multiplicative in $m, n$ if $f(m, n) f\left(m^{\prime}, n^{\prime}\right)=$ $f\left(m m^{\prime}, n n^{\prime}\right)$ whenever $\operatorname{gcd}\left(m n, m^{\prime} n^{\prime}\right)=1$. We see that

$$
\begin{aligned}
M^{(\alpha)}\left(m m^{\prime}, n n^{\prime}\right) & =\sum_{r \mid \operatorname{gcd}\left(m m^{\prime}, n n^{\prime}\right)} r \mu_{\alpha}\left(\frac{m m^{\prime}}{r}\right) \mu_{\alpha-1}\left(\frac{n n^{\prime}}{r}\right) \\
& =\sum_{\substack{r\left|\operatorname{gcd}(m, n) \\
r^{\prime}\right| \operatorname{gcd}\left(m^{\prime}, n^{\prime}\right)}} r r^{\prime} \mu_{\alpha}\left(\frac{m}{r}\right) \mu_{\alpha}\left(\frac{m^{\prime}}{r^{\prime}}\right) \mu_{\alpha-1}\left(\frac{n}{r}\right) \mu_{\alpha-1}\left(\frac{n^{\prime}}{r^{\prime}}\right) \\
& =M^{(\alpha)}(m, n) M^{(\alpha)}\left(m^{\prime}, n^{\prime}\right) .
\end{aligned}
$$

THeOREM 8.2. Let $f$ be a nonzero multiplicative function whose associated Dirichlet series is $F(s)=\sum_{n \geq 1}\left(f(n) / n^{s}\right)$. Let $c^{(\alpha)}(m, n)$ be the GRS of order $\alpha \in \mathbb{C}$.

(i) If $f$ is completely multiplicative, then for each $m \in \mathbb{N}$,

$$
F(s) \sum_{n \geq 1} \frac{f(n) c^{(\alpha)}(m, n)}{n^{s}}=\sum_{n \geq 1} \frac{c^{(\alpha-1)}(m, n)}{n^{s}} f(n) .
$$

(ii) If (8.34) holds for all $m \in \mathbb{N}$, then $f$ is completely multiplicative.

Proof. (i) Assume that $f$ is completely multiplicative. Then

$$
\begin{aligned}
F(s) \sum_{n \geq 1} \frac{f(n) c^{(\alpha)}(m, n)}{n^{s}} & =\sum_{n \geq 1} \frac{1}{n^{s}} \sum_{d \mid n} f(d) c^{(\alpha)}(m, d) f\left(\frac{n}{d}\right) \\
& =\sum_{n \geq 1} \frac{f(n)}{n^{s}} \sum_{d \mid n} c^{(\alpha)}(m, d)=\sum_{n \geq 1} \frac{c^{(\alpha-1)}(m, n)}{n^{s}} f(n)
\end{aligned}
$$

(ii) Assume that (8.34) holds for all $m \in \mathbb{N}$. For each prime $p$ and for each integer $a \geq 2$, it is enough to show that $f\left(p^{a}\right)=f(p)^{a}$.

We first treat the case $a=2$. If $\alpha=0$, putting $m=p$, and equating the coefficients at $n=p^{2}$ in (8.34), we get

$$
\sum_{d \mid p^{2}} f(d) c^{(\alpha)}(p, d) f\left(\frac{p^{2}}{d}\right)=f\left(p^{2}\right) c^{(\alpha-1)}\left(p, p^{2}\right)
$$

Solving this equation gives $f(p)^{2}=f\left(p^{2}\right)$. If $\alpha \neq 0$, putting $m=1$, and equating the coefficients at $n$ in (8.34), we get

$$
\sum_{d \mid n} f(d) \mu_{\alpha}(n) f\left(\frac{n}{d}\right)=f(n) \mu_{\alpha-1}(n) \quad(n \in \mathbb{N})
$$


Substituting $n=p^{2}$ into (8.37), we get

$$
f(1) \mu_{\alpha}(1) f\left(p^{2}\right)+f(p) \mu_{\alpha}(p) f(p)+f\left(p^{2}\right) \mu_{\alpha}\left(p^{2}\right) f(1)=f\left(p^{2}\right) \mu_{\alpha-1}\left(p^{2}\right)
$$

Solving this equation gives $\alpha f\left(p^{2}\right)=\alpha f(p)^{2}$. Since $\alpha \neq 0$, we have $f\left(p^{2}\right)=f(p)^{2}$.

Now consider $a \geq 2$. We proceed by induction. Assuming that $f\left(p^{j}\right)=f(p)^{j}$ for all $1 \leq j \leq a-1$ and substituting $n=p^{a}$ in (8.37), we get

$$
f(1) \mu_{\alpha}(1) f\left(p^{a}\right)+f(p) \mu_{\alpha}(p) f\left(p^{a-1}\right)+\cdots+f\left(p^{a}\right) \mu_{\alpha}\left(p^{a}\right) f(1)=f\left(p^{a}\right) \mu_{\alpha-1}\left(p^{a}\right)
$$

which after simplification leads to

$$
f\left(p^{a}\right)\left[\left(\begin{array}{l}
\alpha-1 \\
a-1
\end{array}\right)-(-1)^{a-1}\right]=f(p)^{a}\left[\left(\begin{array}{l}
\alpha-1 \\
a-1
\end{array}\right)-(-1)^{a-1}\right] .
$$

If $\left(\begin{array}{c}\alpha-1 \\ a-1\end{array}\right) \neq(-1)^{a-1}$, then $f\left(p^{a}\right)=f(p)^{a}$.

If $\left(\begin{array}{c}\alpha-1 \\ a-1\end{array}\right)=(-1)^{a-1}$, then both $\alpha-a+1$ and $a-1$ are nonzero. Consequently, $\left(\begin{array}{l}\alpha-1 \\ a-2\end{array}\right)=$ $(-1)^{a-1}(a-1) /(\alpha-a+1) \neq 0$. Putting $m=p$, and equating the coefficients at $n=p^{a}$ in (8.34) yields

$$
f\left(p^{a}\right) c^{(\alpha-1)}\left(p, p^{a}\right)=\sum_{d \mid p^{a}} f(d) c^{(\alpha)}(p, d) f\left(\frac{p^{a}}{d}\right) .
$$

Solving this equation yields

$$
\begin{aligned}
f\left(p^{a}\right) & \left\{(-1)^{a-1}\left(\begin{array}{l}
\alpha-1 \\
a-1
\end{array}\right)+(-1)^{a-2}\left(\begin{array}{l}
\alpha-1 \\
a-2
\end{array}\right) p-1\right\} \\
= & f(p)^{a}\left\{(-1)^{a-1}\left(\begin{array}{l}
\alpha-1 \\
a-1
\end{array}\right)+(-1)^{a-2}\left(\begin{array}{l}
\alpha-1 \\
a-2
\end{array}\right) p-1\right\} .
\end{aligned}
$$

Since $\left(\begin{array}{l}\alpha-1 \\ a-1\end{array}\right)=(-1)^{a-1}$, and $\left(\begin{array}{l}\alpha-1 \\ a-2\end{array}\right) \neq 0$, we get $f\left(p^{a}\right)=f(p)^{a}$, and the proof is complete.

Now we extend [12, Theorem 2], which gives a relation between Ramanujan sums and generalized Mangoldt functions; but note first that for $m \in \mathbb{N}$ and $\alpha \in \mathbb{C} \backslash\{0\}$, we have the asymptotic relation

$$
c^{(\alpha)}(n, m)=O_{m}(1) \quad(n \longrightarrow \infty),
$$

which follows at once from $\left|c^{(\alpha)}(n, m)\right| \leq \sum_{k(\bmod m)}\left|\mu_{\alpha-1}(\operatorname{gcd}(k, m))\right|$. 
Theorem 8.3. For a fixed $\alpha \in \mathbb{C}$, and $m, n, k \in \mathbb{N}$, if

$$
\begin{array}{r}
M^{(\alpha)}(m, n):=\sum_{r \mid \operatorname{gcd}(m, n)} r \mu_{\alpha}\left(\frac{m}{r}\right) \mu_{\alpha-1}\left(\frac{n}{r}\right), \\
g_{k}^{(\alpha)}(n):=\frac{1}{n} \sum_{r \geq 1} \frac{\mu_{\alpha}(r)}{r}(\log (n r))^{k},
\end{array}
$$

then

$$
\sum_{n \geq 1} \frac{M^{(\alpha)}(m, n)}{n}(\log (n))^{k}=\sum_{n \geq 1} c^{(\alpha)}(n, m) g_{k}^{(\alpha)}(n) .
$$

Proof. Putting $f=\zeta_{0}$ in (8.1) and differentiating (with respect to $s$ ) $k$ times, which is valid by the above asymptotic formula, we get

$$
\begin{aligned}
\sum_{n=1}^{\infty} \frac{M^{(\alpha)}(m, n)}{n^{s}}(\log (n))^{k} & =\sum_{i=0}^{k}\left(\begin{array}{c}
k \\
i
\end{array}\right) \sum_{n=1}^{\infty} \frac{c^{(\alpha)}(n, m)}{n^{s}}(\log (n))^{k-i} \sum_{r=1}^{\infty} \frac{\mu_{\alpha}(r)}{r^{s}}(\log (r))^{i} \\
& =\sum_{n=1}^{\infty} \frac{c^{(\alpha)}(n, m)}{n^{s}} \sum_{r=1}^{\infty}\left(\sum_{i=0}^{k}\left(\begin{array}{c}
k \\
i
\end{array}\right)(\log (n))^{k-i}(\log (r))^{i}\right) \frac{\mu_{\alpha}(r)}{r} \\
& =\sum_{n=1}^{\infty} \frac{c^{(\alpha)}(n, m)}{n^{s}} \sum_{r=1}^{\infty} \frac{\mu_{\alpha}(r)}{r}(\log (n r))^{k} .
\end{aligned}
$$

The desired result follows by letting $s \rightarrow 1^{+}$.

Putting $\alpha=1$, we obtain [12, Theorem 2],

$$
\sum_{n=1}^{\infty} c(n, m) g_{k}^{(1)}(n)=\sum_{n=1}^{\infty} \frac{M^{(1)}(m, n)}{n}(\log (n))^{k}=\sum_{d \mid m} \mu\left(\frac{m}{d}\right)(\log (d))^{k} .
$$

Our last application deals with solutions of certain congruences. When $n, s, r \in \mathbb{N}$, let $N(n, r, s)$ be the number of solutions $\left(x_{1}, \ldots, x_{s}\right) \in \mathbb{Z}^{s}$ of the congruence $n \equiv x_{1}+\cdots+$ $x_{s}(\bmod r)$ subject to the conditions $\operatorname{gcd}\left(x_{i}, r\right)=1(i=1, \ldots, s)$. It is well known [15, Chapter 3] that $N(n, r, s)$ is an even function $(\bmod r)$ in the variable $n$ and $N(n, r, s)=$ $(1 / r) \sum_{d \mid r} c(r / d, r)^{s} c(n, d)$. We end this paper by indicating another connection between a GRS and the number of solutions of certain congruences discovered in [24]. Let $a=$ $\left(a_{1}, a_{2}, \ldots, a_{k}\right) \in \mathbb{Z}^{k}$. Then, for every prime $p$, there uniquely exists an $r \times k$ matrix $B_{p}(a)$ over $\mathbb{Z}_{p}:=\{0,1, \ldots, p-1\}$ such that

$$
a \equiv\left(1, p, \ldots, p^{r-1}\right) B_{p}(a) \quad\left(\bmod p^{r}\right) .
$$

Let $n, k \in \mathbb{N}$ and $a=\left(a_{1}, a_{2}, \ldots, a_{k}\right) \in \mathbb{Z}^{k}$. Then $a$ is said to be $r$ th degree prime to $n$, written as $(a, n)_{r}=1$, if no row vector of $B_{p}(a)$ is a zero-vector for each prime divisor $p$ of $n$. 
Let $N_{r, k}(n)$ denote the number of $k$-vectors $a=\left(a_{1}, a_{2}, \ldots, a_{k}\right) \in \mathbb{Z}_{n}^{k}$ such that $(a, n)_{r}=1$. The following proposition is [24, Theorem 2.2].

Proposition 8.4. If $\alpha, n, k \in \mathbb{N}$, then $N_{\alpha, k}(n)=\phi_{k}^{(\alpha)}(n)$. In particular, if $n$ is $\alpha$-powerful, then $N_{\alpha, k}(n)=\phi_{k}^{(\alpha)}(n)=n^{k} \prod_{p \mid n}\left(1-p^{-k}\right)^{\alpha}$.

The connection with a GRS can be seen for example by observing from Section 2 that if $k=1$, then $c^{(\alpha)}(n, r)=\phi_{1}^{(\alpha)}(r)=N_{\alpha, 1}(r)$, whenever $r \mid n$.

As for the general case, for $p$ prime, let $A_{p}$ be a subset of the $k$-dimensional vector space $\mathbb{Z}_{p}^{k}$. Then there uniquely exists a completely multiplicative function $f$ with $f(p)$ being defined by the number of vectors in $A_{p}$. For any $k$-vector $a(\bmod n)$, we write $(a, n)_{A}=1$ if no row vector of $B_{p}(a)$ is in $A_{p}$, for every prime divisor $p$ of $n$. The following proposition is [24, Theorem 2.3].

Proposition 8.5. If $\alpha, n, k \in \mathbb{N}$ and $n$ is $\alpha$-powerful, then $\phi_{k}^{(\alpha, f)}:=\zeta_{k} * f^{-\alpha}$ just counts the number of all $k$-vectors $a=\left(a_{1}, \ldots, a_{k}\right)(\bmod n)$ such that $(a, n)_{A}=1$.

\section{Acknowledgment}

This work was partially supported by the University of the Thai Chamber of Commerce.

\section{References}

[1] D. R. Anderson and T. M. Apostol, The evaluation of Ramanujan's sum and generalizations, Duke Mathematical Journal 20 (1953), no. 2, 211-216.

[2] T. M. Apostol, Introduction to Analytic Number Theory, Undergraduate Texts in Mathematics, Springer, New York, 1976.

[3] T. C. Brown, L. C. Hsu, J. Wang, and P. J.-S. Shiue, On a certain kind of generalized numbertheoretical Möbius function, The Mathematical Scientist 25 (2000), no. 2, 72-77.

[4] E. Cohen, Representations of even functions $(\bmod r)$. II. Cauchy products, Duke Mathematical Journal 26 (1959), no. 2, 165-182.

[5] P. Erdös and A. Rényi, On the mean value of nonnegative multiplicative number-theoretical functions, The Michigan Mathematical Journal 12 (1965), no. 3, 321-338.

[6] P. Haukkanen, Classical arithmetical identities involving a generalization of Ramanujan's sum, Annales Academiae Scientiarum Fennicae. Series A I. Mathematica Dissertationes (1988), no. 68, 69 pages.

[7] On Generalizations of some identities of Ramanujan, Bulletin de la Société Mathématique de Belgique. Série B 43 (1991), no. 1, 43-57.

[8] _ On the real powers of completely multiplicative arithmetical functions, Nieuw Archief voor Wiskunde. Vierde Serie 15 (1997), no. 1-2, 73-77.

[9] _ The Dirichlet inverse of Ramanujan's sum via principal functions, The Journal of the Indian Mathematical Society. New Series 66 (1999), no. 1-4, 61-64.

[10] P. Haukkanen and P. J. McCarthy, Sums of values of even functions, Portugaliae Mathematica 48 (1991), no. 1, 53-66.

[11] P. Haukkanen and J. Wang, High degree analogs of Menon's identity, Indian Journal of Mathematics 39 (1997), no. 1, 37-42.

[12] A. Ivić, A property of Ramanujan's sums concerning totally multiplicative functions, Serija Matematika i Fizika (1977), no. 577-598, 74-78. 
[13] V. Laohakosol and N. Pabhapote, Properties of rational arithmetic functions, International Journal of Mathematics and Mathematical Sciences 2005 (2005), no. 24, 3997-4017.

[14] V. Laohakosol, N. Pabhapote, and N. Wechwiriyakul, Characterizing completely multiplicative functions by generalized Möbius functions, International Journal of Mathematics and Mathematical Sciences 29 (2002), no. 11, 633-639.

[15] P. J. McCarthy, Introduction to Arithmetical Functions, Universitext, Springer, New York, 1985.

[16] N. Pabhapote, V. Laohakosol, and P. Ruengsinsub, Two aspects of generalized Möbius functions: Landau, Brauer-Rademacher identities and dependence, International Journal of Pure and Applied Mathematics 25 (2005), no. 2, 225-243.

[17] J. Popken, On multiplicative arithmetic functions, Studies in Mathematical Analysis and Related Topics: Essays in Honour of G. Pólya, Stanford University Press, California, 1962, pp. 285-293.

[18] K. G. Ramanathan, Multiplicative arithmetic functions, The Journal of the Indian Mathematical Society. New Series 7 (1943), 111-116.

[19] W. Schwarz and J. Spilker, Mean values and Ramanujan expansions of almost even arithmetical functions, Topics in Number Theory (Proceedings of the Colloquium held in Debrecen, 1974), Colloquia Mathematica Societatis János Bolyai, vol. 13, North-Holland, Amsterdam, 1976, pp. 315-357.

[20] H. N. Shapiro and G. H. Sparer, On algebraic independence of Dirichlet series, Communications on Pure and Applied Mathematics 39 (1986), no. 6, 695-745.

[21] R. Sivaramakrishnan, Classical Theory of Arithmetic Functions, Monographs and Textbooks in Pure and Applied Mathematics, vol. 126, Marcel Dekker, New York, 1989.

[22] J.-M. Souriau, Généralisation de certaines formules arithmétiques d'inversion. Applications, Revue Scientific (Rev. Rose Illus.) 82 (1944), 204-211.

[23] L. Tóth, Remarks on generalized Ramanujan sums and even functions, Acta Mathematica. Academiae Paedagogicae Nyíregyháziensis (New Series) 20 (2004), no. 2, 233-238.

[24] J. Wang and L. C. Hsu, On certain generalized Euler-type totients and Möbius-type functios, Dalian University of Technology, China, preprint.

Vichian Laohakosol: Department of Mathematics, Kasetsart University, Bangkok 10900, Thailand E-mail address: fscivil@ku.ac.th

Pattira Ruengsinsub: Department of Mathematics, Kasetsart University, Bangkok 10900, Thailand E-mail address: fscipar@ku.ac.th

Nittiya Pabhapote: Department of Mathematics, University of the Thai Chamber of Commerce, Bangkok 10400, Thailand

E-mail address: nittiya_pab@utcc.ac.th 


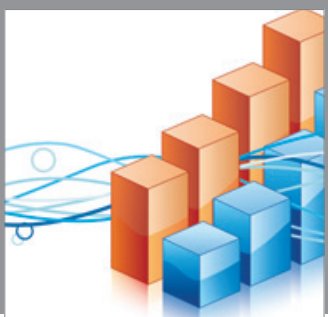

Advances in

Operations Research

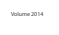

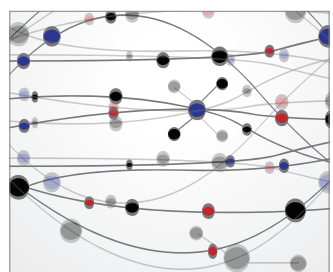

\section{The Scientific} World Journal
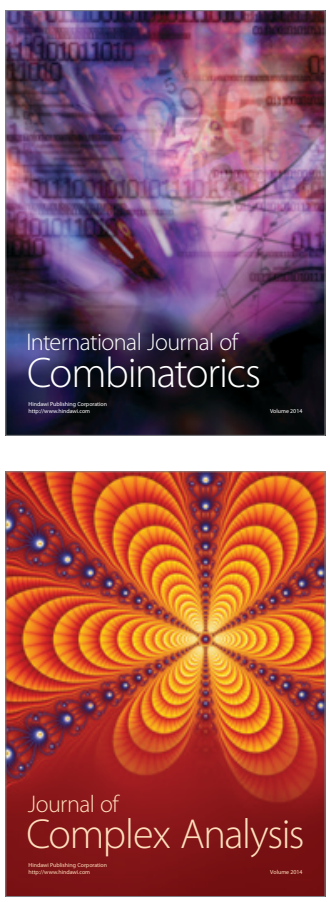

International Journal of

Mathematics and

Mathematical

Sciences
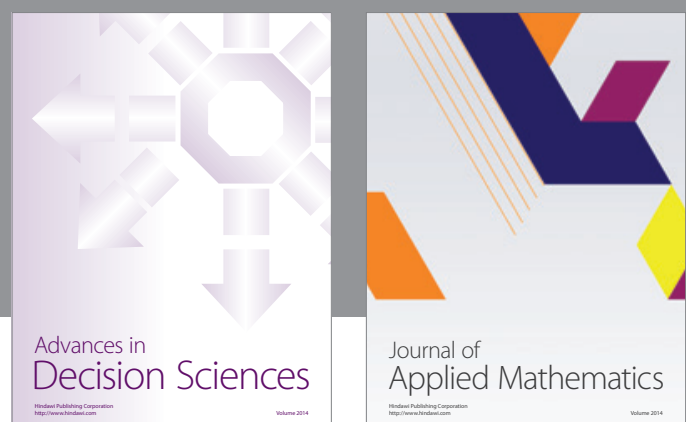

Journal of

Applied Mathematics
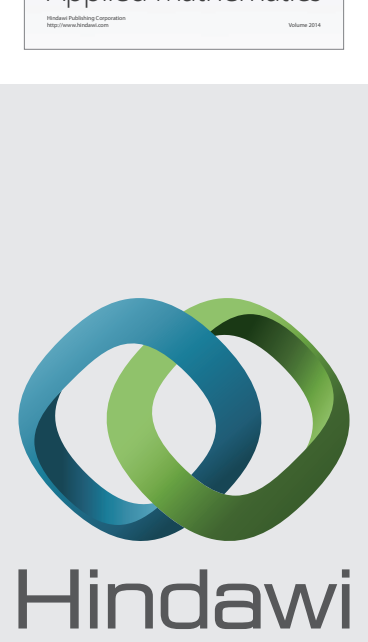

Submit your manuscripts at http://www.hindawi.com
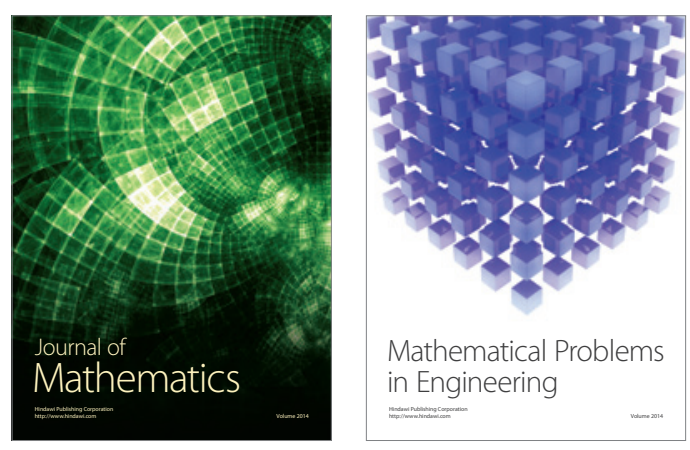

Mathematical Problems in Engineering
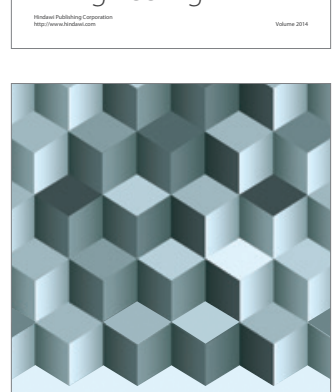

Journal of

Function Spaces
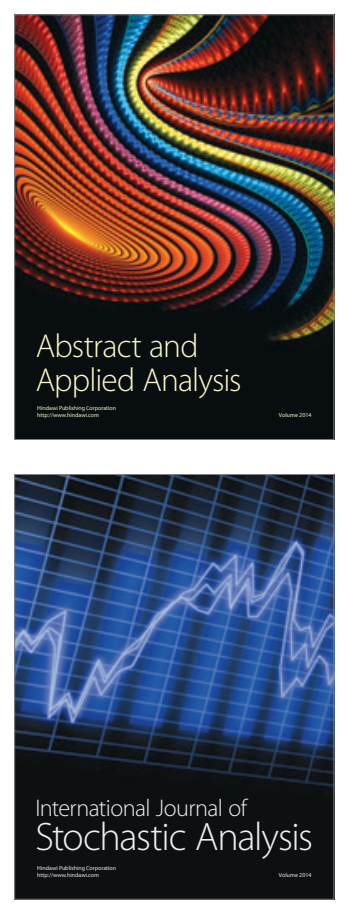

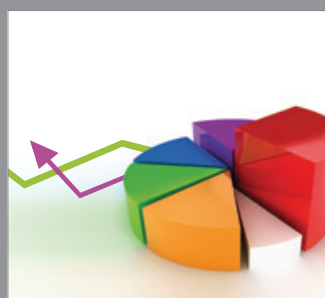

ournal of

Probability and Statistics

Promensencen
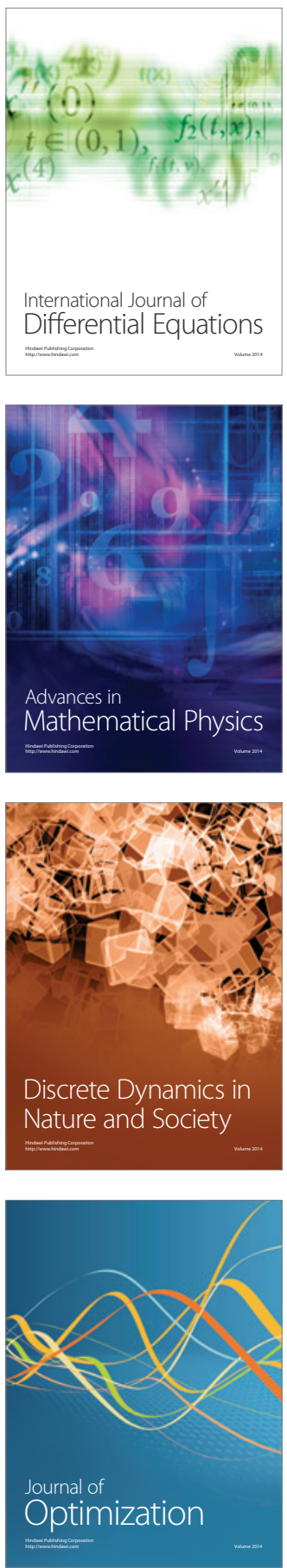